\title{
Pseudodifferential Methods for Boundary Value Problems
}

\author{
Charles L. Epstein \\ Department of Mathematics \\ University of Pennsylvania \\ Philadelphia, PA \\ cle@math. upenn. edu
}

\begin{abstract}
In these lecture notes we introduce some of the concepts and results from microlocal analysis used in the analysis of boundary value problems for elliptic differential operators, with a special emphasis on Dirac-like operators. We first consider the problem of finding elliptic boundary conditions for the $\bar{\partial}$ operator on the unit disk. The rather explicit results in this special case delineate the route we follow for general first order elliptic systems on manifolds with boundary. After some geometric and functional analytic preliminaries, needed to do analysis on manifolds with boundary, we define and describe pseudodifferential operators satisfying the transmission condition. These operators behave well on data with support in a compact subset with a smooth boundary, and include the fundamental solutions of elliptic differential operators. Using the fundamental solution, we define the Calderon projector and establish its basic properties. We then consider boundary conditions defined by pseudodifferential projections, and find a simple criterion for such a boundary operator to define a Fredholm problem. This includes standard elliptic boundary conditions, as well as certain subelliptic problems. A formula is given for the index of such a boundary value problem in terms of the relative index between projectors on the boundary.
\end{abstract}

1991 Mathematics Subject Classification. 58J32, 30E125, 35F15, 35J55, 47A53; Keywords: elliptic operator, boundary conditions, boundary layer method, Fredholm problem.

This version corrects an error in the published version, [11], regarding the distinction between an operator having a compact resolvent, and having the unit ball in the domain of the operator, with respect to the graph norm, compact in $L^{2}$.

Research partially supported by NSF grant DMS06-03973 and the Francis J. Carey term chair. 


\section{CONTENTS}

Introduction $\quad 2$

1. The Basic Example 3

2. Manifolds with Boundary 9

3. Function Spaces on Manifolds with Boundary 10

4. Estimates for Operators Satisfying the Transmission Condition 13

5. The Calderon Projection 20

6. Fredholm Boundary Value Problems for First Order Operators 23

References $\quad 29$

\section{Introduction}

These notes provide an outline for lectures delivered by the author at the Fields Institute on December 13, 2006. The topic of the lectures is the application of pseudodifferential operator techniques to solve boundary value problems for first order differential operators. These techniques have their origins in the single and double layer potential techniques introduced to solve the classical Dirichlet and Neumann problems for the Laplace operator. In the late nineteenth and early twentieth centuries, this subject was called potential theory. The emphasis was on the analysis of the mapping properties of operators defined by convolution with the fundamental solution. A classical treatment of this approach is given in [16], a more modern treatment in [20]. These analytic techniques were the origin of the theory of singular integral operators, which is, in turn, one of the sources of the theory of pseudodifferential operators. In their latter manifestation, these methods came to the fore in the analysis of boundary value problems for the Dirac operator on a manifold with boundary, see $[19,1,2,3,5]$. The boundary conditions we consider are defined by pseudodifferential operators, frequently specialized to pseudodifferential projections. The common theme throughout is the reduction of a boundary value problem to the solution of a pseudodifferential equation on the boundary itself.

We assume a familiarity with the basics of functional analysis, including the theory of $L^{2}$-Sobolev spaces, and elementary aspects of the theory of pseudodifferential operators. Since it is the principal topic of these lectures, we recall the definition and basic properties of Fredholm operators:

Definition 1 Let $X, Y$ be Banach spaces and $A: X \rightarrow Y$ a bounded linear operator. An operator, $A$, is a Fredholm operator provided:

1. $\operatorname{ker} A$ is finite dimensional.

2. $\operatorname{Im} A$ is a closed subspace of $Y$.

3. coker $A=Y / A X$ is finite dimensional.

It is important to recall that if a bounded linear operator has a range of finite codimension, then its range is automatically closed. The fundamental invariant of a Fredholm operator is its index, which is defined by

$$
\operatorname{Ind}(A)=\operatorname{dim} \operatorname{ker} A-\operatorname{dim} \operatorname{coker} A .
$$

If $A: X \rightarrow Y$ is a unbounded operator, then the domain of $A, \operatorname{Dom}(A)$, is generally not all of $X$. If $A$ is a closed operator, then $\operatorname{Dom}(A)$ is a Banach space with respect to the graph norm:

$$
\|x\|_{A}^{2}=\|x\|_{X}^{2}+\|A x\|_{Y}^{2} .
$$


If $X$ and $Y$ are Hilbert spaces, then, with respect to this norm, the graph is as well. An unbounded operator is Fredholm provided, $A:\left(\operatorname{Dom}(A),\|\cdot\|_{A}\right) \rightarrow\left(Y,\|\cdot\|_{Y}\right)$ is a Fredholm operator.

A useful criterion for an operator to be Fredholm is the existence of an almost inverse:

Proposition 1 A necessary and sufficient condition for $A: X \rightarrow Y$ to be Fredholm is the existence of an operator $B: Y \rightarrow X$, such that the differences

$$
K_{1}=\operatorname{Id}_{X}-B A \quad K_{2}=\operatorname{Id}_{Y}-A B
$$

are compact operators.

A parametrix for an elliptic pseudodifferential operator on a compact manifold provides just such an almost inverse.

If the error terms, $K_{1}, K_{2}$, are trace class operators, then there is a very useful formula for the index:

$$
\operatorname{Ind}(A)=\operatorname{tr} K_{1}-\operatorname{tr} K_{2} .
$$

Proofs of these results and many other facts about Fredholm operators can be found in [17].

\section{The Basic Example}

Before going on, we consider, in detail, a simple case, which reveals the main ideas needed to treat the general case. We let $\Omega=D_{1}$, the unit disk in the complex plane. The operator we study is the $\bar{\partial}$-operator,

$$
\bar{\partial} u=\frac{1}{2}\left(\partial_{x}+i \partial_{y}\right) u
$$

The Cauchy-Pompieu formula states that, if $u \in \mathscr{C}^{1}(\bar{\Omega})$, then

$$
u(z)=\frac{1}{\pi} \int_{D_{1}} \frac{\bar{\partial} u(w, \bar{w}) d x d y}{w-z}+\frac{1}{2 \pi i} \int_{b D_{1}} \frac{u(w, \bar{w}) d w}{w-z} .
$$

From the perspective of pseudodifferential operators, this follows from the fact that $[\pi(w-$ $z)]^{-1}$ is a fundamental solution for the $\bar{\partial}$-operator,

$$
\bar{\partial} \frac{1}{\pi(w-z)}=\delta(w-z) \text {. }
$$

As we shall see, the first term in (6) defines a bounded map from $H^{s}\left(D_{1}\right) \rightarrow H^{s+1}\left(D_{1}\right)$, for every $s \in \mathbb{R}$. The second term in formula (6) defines an element of the nullspace of $\bar{\partial}$, that is a holomorphic function, in the complement of $b D_{1}$, The main task before us is to understand the behavior of this second term as $z \rightarrow b D_{1}$.

Using the Fourier representation

$$
u(r, \theta)=\sum_{n=-\infty} u_{n}(r) e^{i n \theta}
$$

we see that

$$
\|u\|_{L^{2}}^{2}=2 \pi \sum_{n=-\infty}^{\infty} \int_{0}^{1}\left|u_{n}(r)\right|^{2} r d r
$$

and, after integrating by parts, we find that

$$
\|\bar{\partial} u\|_{L^{2}}^{2}=\frac{\pi}{2}\left[\sum_{n=-\infty}^{\infty}\left(r\left|a_{n}^{\prime}(r)\right|^{2}+\frac{n^{2}\left|a_{n}(r)\right|^{2}}{r}\right) d r-\sum_{n=-\infty}^{\infty} n\left|a_{n}(1)\right|^{2}\right] .
$$


Our goal is to find boundary conditions for the $\bar{\partial}$-operator, so that the resultant unbounded operator on $L^{2}\left(D_{1}\right)$ is Fredholm and such that the unit ball in the domain of the operator, with respect to the graph norm, is compact in $L^{2}$. If the resolvent set is non-empty, then this implies that the operator has a compact resolvent.

For non-negative integers, define $H^{k}\left(D_{1}\right)$ to be the closure of $\mathscr{C}^{\infty}\left(D_{1}\right)$ with respect to the norm:

$$
\|u\|_{H^{k}}^{2}=\sum_{m+n \leq k}\left\|\partial_{x}^{m} \partial_{y}^{n} u\right\|_{L^{2}\left(D_{1}\right)}^{2} .
$$

For real $s \geq 0$, define $H^{s}\left(D_{1}\right)$ by interpolation. For real $s$, a distribution $u$ in $\mathscr{C}^{-\infty}\left(b D_{1}\right)$ belongs to $H^{s}\left(b D_{1}\right)$ provided:

$$
\|u\|_{H^{s}\left(b D_{1}\right)}^{2}=\sum_{n=-\infty}^{\infty}|\hat{u}(n)|^{2}\left(1+n^{2}\right)^{s}<\infty
$$

where $\hat{u}(n)=\left\langle u, e^{i n \theta}\right\rangle$.

It is not difficult to show that the first two terms on the r.h.s of (10) define a norm equivalent to $\|\nabla u\|_{L^{2}\left(D_{1}\right)}$. A boundary condition for $\bar{\partial}$ defines a Fredholm operator (with the unit ball in the domain of the operator, with respect to the graph norm, compact in $L^{2} D$ ) provided that functions in the domain of the operator satisfy an estimate of the form

$$
\|u\|_{H^{s}\left(D_{1}\right)} \leq C\left[\|\bar{\partial} u\|_{L^{2}\left(D_{1}\right)}+\|u\|_{L^{2}\left(D_{1}\right)}\right]
$$

for an $s>0$. Equation (10) shows that the difficulty in proving this estimate results from the boundary terms $\left\{a_{n}(1): n>0\right\}$, in (10). All other terms on the right hand side of (10) are positive. Indeed if $\bar{\partial} u=0$, then

$$
u(r, \theta)=\sum_{n=0}^{\infty} u_{n} r^{n} e^{i n \theta} .
$$

In this case the negative boundary term in (10) exactly balances the other two positive terms.

While it is not immediate from (10), an $L^{2}$-function $u$ such that $f=\bar{\partial} u \in L^{2}\left(D_{1}\right)$, has an important "global" regularity property. Standard interior estimates imply that $u \in$ $H_{\text {loc }}^{1}\left(D_{1}\right)$, and hence has a well defined restriction to $b D_{r}$, for each $r<1$. Suppose that $\varphi \in \mathscr{C}^{\infty}\left(\overline{D_{1}}\right)$, then a simple integration by parts shows that, for $r<1$, we have:

$$
\int_{b D_{r}} u\left(r, e^{i \theta}\right) \varphi\left(r, e^{i \theta}\right) d z=-2 i\left[\int_{D_{r}} f \varphi d x d y+\int_{D_{r}} u \bar{\partial} \varphi d x d y\right] .
$$

As $u$ and $f$ are in $L^{2}\left(D_{1}\right)$, the limit, as $r \rightarrow 1$, certainly exists on the right hand side of (15), and therefore, the left hand side also has a well defined limit.

Clearly, the limiting pairing on the left hand side of (15) only depends on $\varphi\left\lceil_{b D_{1}}\right.$, hence we can set

$$
\varphi=\sum_{n=0}^{\infty} a_{n} \bar{z}^{n}
$$

The Cauchy-Schwarz inequality then shows that

$$
\left|\sum_{n=1}^{\infty} u_{n}(1) a_{n+1}\right| \leq\|f\|_{L^{2}} \sqrt{\sum_{n=0}^{\infty} \frac{\left|a_{n}\right|^{2}}{2(n+1)}}+\|u\|_{L^{2}} \sqrt{\sum_{n=0}^{\infty} \frac{n\left|a_{n}\right|^{2}}{2}} .
$$

This estimate proves the following basic result: 
Theorem 1 Suppose that $u$ and $\bar{\partial} u$ are in $L^{2}\left(D_{1}\right)$, then $r \mapsto u(r, \cdot)$, is continuous as a map from $(0,1]$ to $H^{-\frac{1}{2}}\left(b D_{r}\right)$. More explicitly,

$$
\sum_{n=-\infty}^{\infty} \frac{\left|u_{n}(r)\right|^{2}}{\sqrt{1+n^{2}}}
$$

is uniformly bounded for $r \in(0,1]$, and

$$
\lim _{r \rightarrow 1^{-}} \sum_{n=-\infty}^{\infty} \frac{\left|u_{n}(r)-u_{n}(1)\right|^{2}}{\sqrt{1+n^{2}}}=0
$$

In other words $u$ has distributional boundary values in the negative order Sobolev space, $H^{-\frac{1}{2}}\left(b D_{1}\right)$. As a corollary we can also use the Cauchy-Pompieu formula for data of this type. This leads naturally to the question: in what sense does the limit

$$
\lim _{z \rightarrow b D_{1}} \frac{1}{2 \pi i} \int_{b D_{1}} \frac{u\left(1, e^{i \theta}\right) d e^{i \theta}}{e^{i \theta}-z}
$$

exist? For the case at hand this question can be answered by a direct calculation. For $z \in D_{1}$, the Cauchy kernel can expanded to give

$$
\frac{1}{e^{i \theta}-z}=e^{-i \theta} \sum_{n=0}^{\infty}\left(e^{-i \theta} z\right)^{n}
$$

Using the expansion in equation (21) we deduce that

$$
\lim _{r \rightarrow 1^{-}} \int_{b D_{1}} \frac{u\left(1, e^{i \theta}\right) d\left(e^{i \theta}\right)}{e^{i \theta}-r e^{i \phi}}=\sum_{n=0}^{\infty} u_{n}(1) e^{i n \phi} .
$$

Indeed, if $u(1, \cdot) \in H^{s}\left(b D_{1}\right)$ for any $s \in \mathbb{R}$, then this limit exists in $H^{s}\left(b D_{1}\right)$.

We denote the projection operator defined on the right hand side of (22) by $\Pi_{+}$. As we shall see, this operator is a pseudodifferential operator of degree zero. For the moment, we compute its principal symbol:

$$
\sigma_{0}\left(\Pi_{+}\right)\left(e^{i \theta}, \xi\right)=\left\{\begin{array}{l}
1 \text { if } \xi>0 \\
0 \text { if } \xi<0 .
\end{array}\right.
$$

To see this, we use oscillatory testing: choose $\phi, \psi$ smooth with compact support, so that $\psi(x)=1$, and $d \phi(x)=\xi$, then

$$
\sigma_{0}(Q)(x, \xi)=\lim _{\lambda \rightarrow \infty} e^{-i \lambda \phi} Q\left(\psi e^{i \lambda \phi}\right)(x) .
$$

For the case at hand, let $\phi_{ \pm}= \pm \theta$, and choose $\psi$ with $\psi\left(e^{i \theta_{0}}\right)=1$, then

$$
\lim _{n \rightarrow \infty} e^{-i n \phi_{ \pm}} \Pi_{+}\left(\psi e^{i n \phi_{ \pm}}\right)\left(e^{i \theta_{0}}\right)=\left\{\begin{array}{l}
\lim _{n \rightarrow \infty} \sum_{j=-n}^{\infty} \psi_{j} e^{i j \theta_{0}}=\psi\left(e^{i \theta_{0}}\right)(+) \\
\lim _{n \rightarrow \infty} \sum_{j=n}^{\infty} \psi_{j} e^{i j \theta_{0}}=0
\end{array}\right.
$$

The operator $\Pi_{+}$is usually called the Cauchy, or Szegó projector, though it agrees with what is, more generally, called the Calderon projector for $\bar{\partial}$.

We now define boundary value problems for the $\bar{\partial}$-operator on $D_{1}$. Let $\mathscr{R}$ denote a pseudodifferential projection acting on distributions defined on the boundary. We define an operator $(\bar{\partial}, \mathscr{R})$ as the unbounded operator on $L^{2}\left(D_{1}\right)$ with the domain

$$
\operatorname{Dom}(\bar{\partial}, \mathscr{R})=\left\{u \in L^{2}\left(D_{1}\right): \bar{\partial} u \in L^{2}\left(D_{1}\right) \text { and } \mathscr{R}\left(u\left\lceil_{b D_{1}}\right)=0\right\} .\right.
$$


Recall that pseudodifferential operators act on spaces of distributions, hence Theorem 1 and the fact that $\mathscr{R}$ is a pseudodifferential operator show that the boundary condition makes sense. It is elementary to prove that this is a closed operator. We now compute the formal adjoint of this operator. A function $v$ is in the domain of the $L^{2}$-adjoint if and only if there exists an $f \in L^{2}\left(D_{1}\right)$ so that, for every $u \in \operatorname{Dom}(\bar{\partial}, \mathscr{R})$ we have:

$$
\langle\bar{\partial} u, v\rangle=\langle u, f\rangle
$$

Taking $v \in \mathscr{C}^{\infty}\left(\overline{D_{1}}\right)$ and integrating by parts we see that

$$
\langle\bar{\partial} u, v\rangle-\left\langle u, \bar{\partial}^{*} v\right\rangle=\left\langle u, e^{-i \theta} v\right\rangle_{b D_{1}} .
$$

For $u\left\lceil_{b D_{1}}\right.$ we can take any function of the form $\mathscr{R} g$, where $g \in \mathscr{C}^{\infty}\left(b D_{1}\right)$. Since the boundary term must vanish, for all such $u$, we see that $\left(\operatorname{Id}-\mathscr{R}^{*}\right) e^{-i \theta} v \Upsilon_{b D_{1}}=0$ is necessary as well. Hence the adjoint boundary condition is that defined by the projector Id $-\mathscr{R}^{*}$. We usually suppose that $\mathscr{R}$ is self adjoint, so that is the same as the boundary condition defined by Id $-\mathscr{R}$.

We now give a condition that implies that this is a Fredholm operator. Our condition is expressed in terms of the comparison operator

$$
\mathscr{T}=\mathscr{R} \Pi_{+}+(\mathrm{Id}-\mathscr{R})\left(\mathrm{Id}-\Pi_{+}\right) .
$$

Theorem 2 The operator $(\bar{\partial}, \mathscr{R})$ is a Fredholm operator provided that $\mathcal{T}$ is an elliptic pseudodifferential operator. If the resolvent set is non-empty, then the resolvent is a compact operator.

Proof First suppose that $u$ lies in the nullspace of $(\bar{\partial}, \mathscr{R})$. In this case $\bar{\partial} u=0$ and therefore $\mathscr{T}\left(u\left\lceil_{b D_{1}}\right)=\mathscr{R}\left(u\left\lceil_{b D_{1}}\right)=0\right.\right.$. As $\mathscr{T}$ is an elliptic operator, this shows that $u$ belongs to a finite dimensional space of smooth functions. Thus the nullspace of $(\bar{\partial}, \mathscr{R})$ is finite dimensional and contained in $\mathscr{C}^{\infty}\left(\overline{D_{1}}\right)$.

The key to proving the theorem is to show that the range of the operator has finite codimension and that, for data in the domain, we have an estimate like that in (13). If we let $\mathscr{b}$ denote the operator defined by the Cauchy kernel, then we need two basic estimates: for $s \in \mathbb{R}$, the following operators are bounded

$$
\begin{aligned}
& u \in H^{s}\left(D_{1}\right) \mapsto \mathscr{C} u \in H^{s+1}\left(D_{1}\right) \\
& f \in H^{s}\left(b D_{1}\right) \mapsto \mathscr{C}\left(f \otimes \delta_{\nu}\right) \in H^{s+\frac{1}{2}}\left(D_{1}\right) .
\end{aligned}
$$

Here $\delta_{v}$ is the $\delta$-measure normal to $b D_{1}$. The map from $H^{s}\left(b D_{1}\right)$ to $H^{s+\frac{1}{2}}\left(D_{1}\right)$ is denoted $\mathscr{K}$, and called the Poisson operator. The hypothesis of the theorem implies that there is a pseudodifferential operator, $\mathcal{U}$ of degree 0 so that

$$
\mathscr{T} U=\mathrm{Id}-K_{1}, \quad \text { UT }=\mathrm{Id}-K_{2},
$$

where $K_{1}, K_{2} \in \Psi^{-\infty}\left(b D_{1}\right)$, and have finite rank.

Let $v \in L^{2}\left(D_{1}\right)$ and set

$$
u_{1}=\mathscr{C v} \text { and } u_{0}=-\mathscr{K} U \Re\left(u_{1}\left\lceil_{b D_{1}}\right) .\right.
$$

From the Cauchy-Pompieu formula it follows that, in the sense of distributions, $\bar{\partial}\left(u_{0}+\right.$ $\left.u_{1}\right)=v$. Moreover, the fact that $u \rightarrow u \uparrow_{b D_{1}}$ is bounded from $H^{1}\left(D_{1}\right) \rightarrow H^{\frac{1}{2}}\left(b D_{1}\right)$ and (30) imply that both $u_{0}$, and $u_{1}$ belong to $H^{1}\left(D_{1}\right)$; there is a constant $C$ so that

$$
\left\|u_{0}+u_{1}\right\|_{H^{1}\left(D_{1}\right)} \leq C\|v\|_{L^{2}} .
$$

It remains to check the boundary condition. To that end we state a simple but fundamental lemma. 
Lemma 1 If $\mathscr{T} f \in \operatorname{Im} \Re$, then

$$
\mathscr{T} \Pi_{+} f=\mathscr{T} f .
$$

Proof The proof is an elementary computation: If $\mathscr{T} f \in \operatorname{Im} \mathscr{R}$, then

$$
\mathscr{T} f=\mathscr{R} \mathscr{T} f=\mathscr{T} \Pi_{+} f .
$$

We see that the boundary value of $u_{0}$ is $-\Pi_{+} U \Re\left(u_{1} \uparrow_{b D_{1}}\right)$, and

$$
\mathscr{T} U \Re\left(u_{1} \uparrow_{b D_{1}}\right)=\left(\mathrm{Id}-K_{1}\right) \mathscr{R}\left(u_{1} \uparrow_{b D_{1}}\right) \text {. }
$$

Assume that $v$ is chosen so that

$$
K_{1} \mathscr{R}\left(u_{1} \uparrow_{b D_{1}}\right)=0 ;
$$

this amounts to imposing finitely many, bounded linear conditions. With this assumption we see that

$$
\mathscr{T} U \mathscr{R}\left(u_{1} \Upsilon_{b D_{1}}\right)=\mathscr{R}\left(u_{1}\left\lceil_{b D_{1}}\right) \in \operatorname{Im} \mathscr{R},\right.
$$

hence the lemma implies that

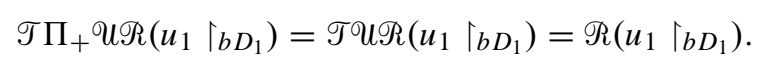

Putting the pieces together, we have shown that, if $v \in L^{2}\left(D_{1}\right)$ satisfies the finitely many linear conditions in (37), then there is a solution $u \in \operatorname{Dom}(\bar{\partial}, \mathscr{R})$ to the equation

$$
\bar{\partial} u=v,
$$

which satisfies $\|u\|_{H^{1}\left(D_{1}\right)} \leq C\|v\|_{L^{2}\left(D_{1}\right)}$. Hence the range of the operator is of finite codimension and therefore closed. The nullspace is finite dimensional and this suffices to show that the operator is Fredholm.

To show that $\operatorname{Dom}(\bar{\partial}, \mathscr{R}) \subset H^{1}\left(D_{1}\right)$, we suppose that $\bar{\partial} u=f, \mathscr{R}\left(u\left\lceil_{b D_{1}}\right)=0\right.$. Let $u_{1}=\mathscr{b} f \in H^{1}\left(D_{1}\right)$. Then $u_{0}=u-u_{1}$ satisfies,

$$
\bar{\partial} u_{0}=0 \text { and } \mathscr{R}\left(u_{0}\left\lceil_{b D_{1}}\right)=-\mathscr{R}\left(u_{1}\left\lceil_{b D_{1}}\right) \in H^{\frac{1}{2}}\left(b D_{1}\right) .\right.\right.
$$

Since $\bar{\partial} u_{0}=0$, we see that

$$
-\mathscr{R}\left(u_{1} \Upsilon_{b D_{1}}\right)=\mathscr{T}\left(u_{0} \Upsilon_{b D_{1}}\right)
$$

and therefore

$$
\left(\mathrm{Id}-K_{2}\right) u_{0} \uparrow_{b D_{1}}=-U \Re\left(u_{1} \Upsilon_{b D_{1}}\right) \in H^{\frac{1}{2}}\left(b D_{1}\right) .
$$

As $K_{2}$ is a smoothing operator, we see that there is a constant $C_{1}$, such that if $u \in$ $\operatorname{Dom}(\bar{\partial}, \mathscr{R})$, then

$$
\|u\|_{H^{1}\left(D_{1}\right)} \leq C_{1}\left[\|\bar{\partial} u\|_{L^{2}\left(D_{1}\right)}+\|u\|_{L^{2}\left(D_{1}\right)}\right] .
$$

This estimate implies that $\operatorname{Dom}(\bar{\partial}, \mathscr{R}) \subset H^{1}\left(D_{1}\right)$, which implies that the unit ball in the domain of the operator, with respect to the graph norm, is compact in $L^{2}$. If the resolvent set is non-empty, then the resolvent is compact.

In fact much more is true: for each $s \in[0, \infty)$, there is a $C_{s}$, so that if $\bar{\partial} u=f \in H^{s}\left(D_{1}\right)$, and $\mathscr{R}\left(u \uparrow_{b D_{1}}\right)=0$, then $u \in H^{s+1}$ and

$$
\|u\|_{H^{s+1}\left(D_{1}\right)} \leq C_{s}\left[\|f\|_{H^{s}\left(D_{1}\right)}+\|u\|_{L^{2}\left(D_{1}\right)}\right] .
$$


As a corollary of this theorem we can identify the $L^{2}$-adjoint of $(\bar{\partial}, \mathscr{R})$ with the operator defined by $\left(\bar{\partial}^{*}, e^{i \theta}(\mathrm{Id}-\mathscr{R}) e^{-i \theta}\right)$. The condition that $\mathscr{T}$ be an elliptic pseudodifferential operator, coupled with the fact that $\mathscr{R}$ is a projection implies that

$$
\sigma_{0}(\mathscr{R})\left(e^{i \theta}, \xi\right)=\left\{\begin{array}{l}
1 \text { if } \xi>0 \\
0 \text { if } \xi<0 .
\end{array}\right.
$$

There are many possible projections satisfying this condition.

These estimates imply a fundamental relationship between the projectors $\mathscr{R}$ and $\Pi_{+}$.

Corollary 1 If $\mathcal{T}$ is an elliptic pseudodifferential operator, then the restriction

$$
\mathscr{R}: \operatorname{Im} \Pi_{+} \longrightarrow \operatorname{Im} \mathscr{R}
$$

is a Fredholm operator. We denote this restriction by $\Re^{\Pi_{+}}$.

Proof The operator $Q=\Pi_{+} U \mathscr{R}$ is a parametrix for $\mathscr{R}^{\Pi_{+}}$:

$$
\begin{aligned}
& Q \mathscr{R} \Pi_{+}=\Pi_{+} \cup \mathscr{T} \Pi_{+}=\Pi_{+}\left(\mathrm{Id}-K_{2}\right) \Pi_{+} \\
& \mathscr{R} \Pi_{+} Q=\mathscr{R} \mathscr{T} \cup \mathscr{R}=\mathscr{R}\left(\mathrm{Id}-K_{1}\right) \mathscr{R} .
\end{aligned}
$$

The conclusion follows from Proposition 1.

Definition 2 The index of $\mathscr{R}^{\Pi_{+}}$is called the relative index of $\left(\Pi_{+}, \mathscr{R}\right)$, denoted $\mathrm{R}-\operatorname{Ind}\left(\Pi_{+}, \mathscr{R}\right)$.

The relative index of the boundary projectors equals the index of the boundary value problem.

Theorem 3 If $\Re$ is a self adjoint projector defining a Fredholm boundary value problem for $\bar{\partial}$, then

$$
\operatorname{Ind}(\bar{\partial}, \mathscr{R})=\operatorname{R}-\operatorname{Ind}\left(\Pi_{+}, \mathscr{R}\right) .
$$

Proof It is easy to see that the nullspace of $(\bar{\partial}, \mathscr{R})$ agrees with that of $\mathscr{R}^{\Pi_{+}}$. A function $u$ in the nullspace of $(\bar{\partial}, \mathscr{R})$ belongs to ker $\bar{\partial}$ and therefore $u\left\lceil_{b D_{1}} \in \operatorname{Im} \Pi_{+}\right.$. The boundary condition, $\mathscr{R} u\left\lceil_{b D_{1}}=0\right.$, shows that $u\left\lceil_{b D_{1}}\right.$ is in the nullspace of $\mathscr{R}^{\Pi_{+}}$. On the other hand, if $f \in \operatorname{Im} \Pi_{+}$, then there is a holomorphic function $u$, with $u\left\lceil_{b D_{1}}=f\right.$. This shows that

$$
\operatorname{ker}(\bar{\partial}, \mathscr{R})=\operatorname{ker} \mathscr{R}^{\Pi_{+}} .
$$

The coker $\mathscr{R}^{\Pi_{+}}$consists of functions $f \in \operatorname{Im} \mathscr{R}$ such that $\left(\mathrm{Id}-\Pi_{+}\right) f=0$. The nullspace of $(\bar{\partial}, \mathscr{R})^{*}$ consists of functions $v$ such that

$$
\bar{\partial}^{*} v=-\partial_{z} v=0 \text { and }(\mathrm{Id}-\mathscr{R}) e^{-i \theta} v\left\lceil_{b D_{1}}=0 .\right.
$$

This implies that $v \in \operatorname{ker}(\bar{\partial}, \mathscr{R})^{*}$ if and only if $\bar{z} v \uparrow_{b D_{1}}$ represents an equivalence class in coker $\mathscr{R}^{\Pi_{+}}$. This completes the proof of the theorem.

Combining this result with (48) and the trace formula, equation (4), we obtain a trace formula for $\operatorname{Ind}(\bar{\partial}, \mathscr{R})$ :

$$
\operatorname{Ind}(\bar{\partial}, \mathscr{R})=\operatorname{tr} \Pi_{+} K_{2} \Pi_{+}-\operatorname{tr} \Re K_{1} \Re .
$$

Remark 1 In Hyunsuk Kang's thesis, [15], a variety of boundary projectors of this type are constructed. She considers the following geometric situation: suppose that $\gamma$ : $S^{1} \rightarrow \mathbb{C}^{k}$ is a smooth, oriented real curve. Let $\mathscr{P}$ denote the $L^{\infty}$-closure of the algebra of polynomials in $\mathbb{C}^{k}$ restricted to $\gamma\left(S^{1}\right)$, and $\mathscr{P}_{\gamma}$ its pullback to $S^{1}$, via $\gamma$. Finally let 
$\mathscr{R}_{\gamma}$ denote the orthogonal projection onto the $L^{2}$-closure of $\mathscr{P}_{\gamma}$. The oriented curve $\gamma\left(S^{1}\right)$ bounds a holomorphic curve $X$ in $\mathbb{C}^{k} \backslash \gamma\left(S^{1}\right)$ if and only if the restriction, $\mathscr{R}_{\gamma}^{\Pi_{+}}$:

$$
\mathscr{R}_{\gamma}: \operatorname{Im} \Pi_{+} \longrightarrow \operatorname{Im} \mathscr{R}_{\gamma}
$$

is a Fredholm operator. In this case, there is a formula for the relative index, $\mathrm{R}-\operatorname{Ind}\left(\Pi_{+}, \mathscr{R}_{\gamma}\right)$, in terms of analytic and geometric invariants of $X$. For example, if $X$ is a smooth holomorphic curve of genus $g$ then

$$
\operatorname{R-Ind}\left(\Pi_{+}, \mathscr{R}_{\gamma}\right)=g
$$

It is clear that the main conclusions of Theorem 2 remain true if there is an $\mu<1$ so that the operator $U: H^{s}\left(b D_{1}\right) \rightarrow H^{s-\mu}\left(b D_{1}\right)$, for all $s \geq-\frac{1}{2}$. In the 1-dimensional case such examples are not naturally occurring, though in higher dimensions they are quite important.

A similar discussion applies to study higher order elliptic equations as well. For example if $P=\Delta=\left(\partial_{x}^{2}+\partial_{y}^{2}\right)$, then $G(x, y)=[2 \pi]^{-1} \log |z-w|$ is a fundamental solution. Green's formula states that, if $u \in \mathscr{C}^{2}\left(\bar{D}_{1}\right)$, then

$$
u(z)=\int_{D_{1}} \Delta u(w) G(z, w) d A_{w}+\int_{b D_{1}}\left[u(w) \partial_{v_{w}} G(z, w)-\partial_{v_{w}} u(w) G(z, w)\right] d s_{w},
$$

here $v$ is the outward unit normal vector to $b D_{1}$. If $\Delta u=0$, then $u$ is determined by its Cauchy data $\left(u, \partial_{v} u\right)\left\lceil_{b D_{1}}\right.$. The Green's function satisfies estimates much like those satisfied by the Cauchy kernel. The Calderon projector, $\mathscr{P}$, takes a pair of functions defined on the boundary $(f, g)$ to the pair $\left(u, \partial_{\nu} u\right) \uparrow_{b D_{1}}$, where $u$ is the element of ker $\Delta$, given by

$$
u(z)=\int_{b D_{1}}\left[f(w) \partial_{v_{w}} G(z, w)-g(w) G(z, w)\right] d s_{w} .
$$

Boundary conditions are now defined by pseudodifferential projections $\mathscr{R}$ acting on the pair $\left(u, \partial_{\nu} u\right)\left\lceil_{b D_{1}}\right.$. The BVP is elliptic if the comparison operator $\mathscr{T}=\mathscr{R} \mathscr{P}+(\operatorname{Id}-\mathscr{R})(\operatorname{Id}-\mathscr{P})$ is elliptic. For simplicity we will largely stick to the case of first order systems in the sequel.

\section{Manifolds with Boundary}

Let $\Omega$ be a closed, $n$-dimensional manifold with boundary. As local models we have

$$
\mathbb{B}_{1}=\left\{x \in \mathbb{R}^{n}:\|x\|<1\right\} \text { and } \mathbb{B}_{1}^{+}=\left\{x \in \mathbb{R}^{n}:\|x\|<1 \text { and } x_{n} \geq 0\right\} .
$$

The interior of $\Omega$ has a cover by open sets $\left\{U_{j}\right\}$ and the boundary has a cover by open sets $\left\{V_{k}\right\}$ such that, for each $j$ there is a homeomorphism $\varphi_{j}: U_{j} \rightarrow \mathbb{B}_{1} \subset \mathbb{R}^{n}$, and for each $k$ there is a homeomorphism $\varphi_{k}: V_{k} \rightarrow \mathbb{B}_{1}^{+} \subset \mathbb{R}_{+}^{n}$. In the later case $\varphi_{k}\left(V_{k} \cap b \Omega\right) \subset b \mathbb{B}_{1}^{+}$. The pairs $\left(U_{j}, \varphi_{j}\right)$ are called interior coordinate charts and $\left(V_{k}, \varphi_{k}\right)$ are boundary coordinate charts. On the nontrivial intersections of the coordinate charts we require that the induced maps from subsets of $\mathbb{R}^{n}$ to subsets of $\mathbb{R}^{n}$ be diffeomorphisms, e.g. If $U_{j} \cap U_{j^{\prime}} \neq \emptyset$, then

$$
\varphi_{j} \circ \varphi_{j^{\prime}}^{-1}: \varphi_{j^{\prime}}\left(U_{j} \cap U_{j^{\prime}}\right) \longrightarrow \varphi_{j}\left(U_{j} \cap U_{j^{\prime}}\right)
$$

is a diffeomorphism.

A function, $r$, which is non-negative (or non-positive) in the interior of $\Omega$ and vanishes to order one $(d r \neq 0)$ along the boundary is called a defining function for the boundary of $\Omega$. The normal bundle to the boundary is the line bundle along the boundary

$$
N b \Omega=T \Omega \uparrow_{b \Omega} / T b \Omega .
$$


The dual bundle, the co-normal bundle, $N^{*} b \Omega$, is the sub-bundle of $T^{*} \Omega\left\lceil_{b \Omega}\right.$ consisting of 1-forms that annihilate $T b \Omega$. It is spanned at every point, $x$ by $d r_{x}$. The geometry of $\Omega$ near to the boundary is described by the tubular neighborhood theorem:

Theorem 4 (The tubular neighborhood theorem) If $\Omega$ is a manifold with boundary, then there is a neighborhood $U$ of $b \Omega$ that is diffeomorphic to $b \Omega \times[0,1)$. It can be realized as a one sided neighborhood of the zero section within $N b \Omega$.

Using the identification of $U$ with a neighborhood of the zero section, it is easy to show that $\Omega$ can be embedded as a subset of the smooth manifold without boundary: $\widetilde{\Omega} \simeq$ $\Omega \amalg_{b \Omega} \Omega$. The interior of $\Omega$ is an open subset of $\widetilde{\Omega}$. If $\Omega$ is a compact manifold with boundary, then $\widetilde{\Omega}$ is a compact manifold without boundary. If we fix an orientation on $\Omega$, then $\widetilde{\Omega} \simeq \Omega \amalg_{b \Omega}[-\Omega]$, where [- $\left.\Omega\right]$ denotes $\Omega$ with the opposite orientation, is also an oriented manifold.

We use $\mathscr{C}^{\infty}(\Omega), \mathscr{C}^{k}(\Omega)$, etc. to denote smooth, respectively $\mathscr{C}^{k}$-functions on the interior of $\Omega$, and $\mathscr{C}^{\infty}(\bar{\Omega}), \mathscr{C}^{k}(\bar{\Omega})$, these classes of functions on the closure. If $F \rightarrow \Omega$ is a vector bundle, then $\mathscr{C}^{\infty}(\Omega ; F), \mathscr{C}^{k}(\Omega ; F)$ are the sections of $F$, that are smooth, respectively. If it is clear from the context, we often omit explicit mention of the bundle from the notation. When doing analysis on a manifold with boundary it is very useful to be able to extend functions from $\bar{\Omega}$ to $\widetilde{\Omega}$. Seeley proved a very general such result:

Theorem 5 (Seeley Extension Theorem) If $\Omega$ is a manifold with boundary, then there is a continuous linear map

$$
E: \mathscr{C}^{\infty}(\bar{\Omega}) \longrightarrow \mathscr{C}^{\infty}(\widetilde{\Omega}) \text {. }
$$

For each $k \in \mathbb{N}_{0}$ there is also a continuous linear map $E_{k}: \mathscr{C}^{k}(\bar{\Omega}) \rightarrow \mathscr{C}^{k}(\widetilde{\Omega})$.

Recall that, for $s \in \mathbb{R}$, the $L^{2}$-Sobolev space $H^{s}\left(\mathbb{R}^{n}\right)$ is defined as those tempered distributions $u \in \mathscr{Y}^{\prime}\left(\mathbb{R}^{n}\right)$ whose Fourier transform $\hat{u}$ is a function, which satisfies:

$$
\|u\|_{s}^{2}=\int_{\mathbb{R}^{n}}|\hat{u}(\xi)|^{2}\left(1+|\xi|^{2}\right)^{s} d \xi<\infty .
$$

Let $X$ be a compact manifold without boundary, having coordinate cover $\left(U_{j}, \varphi_{j}\right)$. Let $\left\{\psi_{j}\right\}$ be a partition of unity subordinate to this cover. A distribution $u \in \mathscr{C}^{-\infty}(X)$ belongs to $H^{s}(X)$, if for every $j$, the compactly supported distribution $\left(\psi_{j} u\right) \circ \varphi_{j}^{-1}$, defined on $\mathbb{R}^{n}$, belongs to $H^{s}\left(\mathbb{R}^{n}\right)$. It is a well known result that the Sobolev spaces are invariant under such changes of coordinate and therefore, the space $H^{s}(X)$ is well defined as a topological vector space. A norm, which defines this topology is given by

$$
\|u\|_{H^{s}(X)}^{2}=\sum_{j}\left\|\left(\psi_{j} u\right) \circ \varphi_{j}^{-1}\right\|_{H^{s}\left(\mathbb{R}^{n}\right)}^{2} .
$$

Defining function spaces on manifolds with boundary is a bit more involved, we return to this question in Section 3

Good references for the material in this section are [14] and [22].

\section{Function Spaces on Manifolds with Boundary}

To extend the results of the previous section to the case of a general manifold with boundary we introduce function spaces that are adapted to the study of boundary value problems. Let $\Omega$ denote a compact manifold with boundary, which we often think of as a subset of its double, $\widetilde{\Omega}$. There is a certain amount of subtlety involved in the definitions of spaces of distributions on a manifold with boundary, which, in the end, has to do with what one means by regularity up to the boundary. We usually think of $\Omega$ as a closed subset of 
$\widetilde{\Omega}$, but in this section we often emphasize that point by writing $\bar{\Omega}$. In general we assume that a smooth metric is fixed on $\widetilde{\Omega}$ and let $\partial_{v}$ denote differentiation with respect to the unit vector field, $v$, normal to $b \Omega$.

The main distinction derives from whether one wishes to consider a function to be smooth on $\Omega$ if the function and all its derivatives extend smoothly to $b \Omega$, or one wishes to consider a function to be smooth on $\Omega$ if the function and all its derivatives vanish along $b \Omega$. In the latter case, its extension by zero to all of $\widetilde{\Omega}$ is smooth. We denote the former space of functions by $\mathscr{C}^{\infty}(\bar{\Omega})$ and the later by $\dot{\mathscr{C}}^{\infty}(\Omega)$. The elements of the dual space of $\mathscr{C}^{\infty}(\bar{\Omega})$ are called supported distributions and are denoted by $\dot{\mathscr{C}}^{-\infty}(\Omega)$. The elements of the dual space of $\dot{\mathscr{C}}^{\infty}(\Omega)$ are called extendible distributions, and are denoted by $\mathscr{C}^{-\infty}(\Omega)$.

An important difference between these two spaces concerns the action of differential operators. As usual this is defined by duality: if $P$ is any differential operator then $P^{t}$ maps both spaces of smooth functions to themselves, and therefore we can define an action of $P$ on either $\dot{\mathscr{C}}^{-\infty}(\Omega)$ or $\mathscr{C}^{-\infty}(\Omega)$ by duality:

$$
\langle P u, \varphi\rangle \stackrel{d}{=}\left\langle u, P^{t} \varphi\right\rangle .
$$

If $u \in \mathscr{C}^{-\infty}(\Omega)$, then we take $\varphi \in \dot{\mathscr{C}}^{\infty}(\Omega)$ in equation (63), while if $u \in \dot{\mathscr{C}}^{-\infty}(\Omega)$, then we take $\varphi \in \mathscr{C}^{\infty}(\bar{\Omega})$. Of course $\mathscr{C}^{\infty}(\bar{\Omega})$ is a subset of both $\dot{\mathscr{C}}^{-\infty}(\Omega)$ and $\mathscr{C}^{-\infty}(\Omega)$. If $u \in \mathscr{C}^{\infty}(\bar{\Omega})$, then the meaning of $P u$ depends on whether we think of it as an extendible or a supported distribution. The difference in the two definitions is a distribution with support on $b \Omega$. For example, if $u \in \mathscr{C}^{\infty}\left(\bar{D}_{1}\right)$ and $P=\bar{\partial}$ then

$$
\bar{\partial}_{\mathrm{ext}} u-\bar{\partial}_{\mathrm{supp}} u=\delta(r-1) \frac{u\left(1, e^{i \theta}\right) e^{i \theta} d \theta}{2} .
$$

A distribution $u \in \dot{\mathscr{C}}^{-\infty}(\Omega)$ if and only if there is an element $U \in \mathscr{C}^{-\infty}(\widetilde{\Omega})$ such that supp $U \subset \bar{\Omega}$, which defines $u$. In this case $u$ is defined on an element $\varphi \in \mathscr{C}^{\infty}(\bar{\Omega})$ by

$$
u(\varphi)=U(\widetilde{\varphi}),
$$

where $\widetilde{\varphi}$ is any extension of $\varphi$ to an element of $\mathscr{C}^{\infty}(\widetilde{\Omega})$, for example the Seeley extension $E \varphi$. Because supp $U \subset \bar{\Omega}$, the value of $U(\widetilde{\varphi})$ is independent of which extension is used. The $H^{s}$-norm is defined on supported distributions by setting

$$
\|u\|_{s}=\|U\|_{H^{s}(\widetilde{\Omega})} .
$$

The subspace of $\dot{\mathscr{C}}^{-\infty}(\Omega)$ for which this norm is finite is denoted by $\dot{H}^{s}(\Omega)$. The important thing to note about this space is that in order for $u$ to be smooth in this sense, that is belonging to $\dot{H}^{s}(\Omega)$, for a large value of $s$, it must have many derivatives in $\Omega$, which vanish at the boundary. This is because supp $U \subset \Omega$. The space $\dot{\mathscr{C}}^{\infty}(\Omega)$ is a dense subset of $\dot{\mathscr{C}}^{-\infty}(\Omega)$.

On the other hand $\dot{\mathscr{C}}^{\infty}(\Omega)$ is a closed subspace of $\mathscr{C}^{\infty}(\widetilde{\Omega})$ and therefore the HahnBanach theorem implies that if $u \in \mathscr{C}^{-\infty}(\Omega)$, then there is $U \in \mathscr{C}^{-\infty}(\widetilde{\Omega})$ that extends $u$. We define the $H^{s}$-norm for this space of distributions by

$$
\|u\|_{s}=\inf _{U \text { extending } u}\|U\|_{H^{s}} .
$$

The subspace of $\mathscr{C}^{-\infty}(\Omega)$ for which this norm is finite is denoted by $H^{s}(\Omega)$. From the definition of the norm, it is again clear that a distribution $u$ is smooth in this sense if it has many derivatives with smooth extensions to $b \Omega$, rather than having to vanish to high order along $b \Omega$. The space $\mathscr{C}^{\infty}(\bar{\Omega})$ is dense in $\mathscr{C}^{-\infty}(\Omega)$. It is clear that for every $s \in \mathbb{R}$, we have a natural map: $\dot{H}^{s}(\Omega) \rightarrow H^{s}(\Omega)$. This map turns out to be injective if $s \geq-\frac{1}{2}$ and 
surjective if $s \leq \frac{1}{2}$. The $L^{2}$-pairing on $\Omega$ between $\mathscr{C}^{\infty}(\bar{\Omega})$ and $\dot{\mathscr{C}}^{\infty}(\Omega)$ can be extended to show that, for all $s \in \mathbb{R}$, we have the isomorphisms

$$
\left[H^{s}(\Omega)\right]^{\prime} \simeq \dot{H}^{-s}(\Omega) \text { and }\left[\dot{H}^{s}(\Omega)\right]^{\prime} \simeq H^{-s}(\Omega) .
$$

If $s>\frac{1}{2}$, then restriction to the boundary extends to define a continuous trace map:

$$
\tau: H^{s}(\Omega) \longrightarrow H^{s-\frac{1}{2}}(b \Omega) .
$$

Because this map is not defined for $s=\frac{1}{2}$, it is convenient to work with spaces that treat regularity in the tangential and normal directions slightly differently. These spaces greatly facilitate the analysis of differential operators defined on $L^{2}(\Omega)$. We first define these spaces for the half space $\mathbb{R}_{+}^{n}$. Let $x^{\prime}=\left(x_{1}, \ldots, x_{n-1}\right), \xi^{\prime}=\left(\xi_{1}, \ldots, \xi_{n-1}\right)$, and define the tangential Fourier transform to be

$$
\widetilde{u}\left(\xi^{\prime}, x_{n}\right)=\int_{\mathbb{R}^{n-1}} u\left(x^{\prime}, x_{n}\right) e^{-i x^{\prime} \cdot \xi^{\prime}} d x^{\prime} .
$$

For $m$ a non-negative integer and $s \in \mathbb{R}$ we define

$$
\|u\|_{(m, s)}^{2}=\sum_{j=0}^{m} \int_{\mathbb{R}^{n-1}} \int_{0}^{\infty}\left|\partial_{x_{n}}^{j} \widetilde{u}\left(\xi^{\prime}, x_{n}\right)\right|^{2}\left(1+\left|\xi^{\prime}\right|^{2}\right)^{s-j} d \xi^{\prime} d x_{n} .
$$

The space $H_{(m, s)}\left(\mathbb{R}_{+}^{n}\right)$ is the closure of $\mathscr{C}_{c}^{\infty}\left(\overline{\mathbb{R}}_{+}^{n}\right)$ with respect to this norm. It consists of all distributions in $\mathscr{C}^{-\infty}\left(\overline{\mathbb{R}_{+}^{n}}\right)$ such that $\partial_{x_{n}}^{j} \widetilde{u}$ is a function for $0 \leq j \leq m$, and the norm in (71) is finite. The corresponding space of supported distributions, $\dot{H}_{(m, s)}\left(\mathbb{R}_{+}^{n}\right)$, is defined as the closure of $\mathscr{C}_{c}^{\infty}$ (int $\mathbb{R}_{+}^{n}$ ) with respect to this norm.

These spaces are useful for two reasons:

Theorem 6 If $m$ is a positive integer and $0 \leq j<m$, then the map

$$
\mathscr{C}^{\infty}\left(\mathbb{R}_{+}^{n}\right) \ni u \rightarrow \partial_{x_{n}}^{j} u\left(\cdot, x_{n}\right),
$$

for $x_{n} \geq 0$, extends as a continuous map from $H_{(m, s)}\left(\mathbb{R}_{+}^{n}\right) \rightarrow H^{s+m-j-\frac{1}{2}}\left(\mathbb{R}^{n-1}\right)$. Moreover, $x_{n} \rightarrow \partial_{x_{n}}^{j} u\left(\cdot, x_{n}\right)$ is continuous from $[0,1)$ to $H^{s+m-j-\frac{1}{2}}\left(\mathbb{R}^{n-1}\right)$.

Of particular note is the fact that $H^{\frac{1}{2}}\left(\mathbb{R}_{+}^{n}\right) \supset H_{\left(1,-\frac{1}{2}\right)}\left(\mathbb{R}_{+}^{n}\right)$. While the restriction to the boundary is not defined for $u \in H^{\frac{1}{2}}\left(\mathbb{R}_{+}^{n}\right)$, it is defined, as an element of $L^{2}\left(b \mathbb{R}_{+}^{n}\right)$, for $u \in H_{\left(1,-\frac{1}{2}\right)}\left(\mathbb{R}_{+}^{n}\right)$.

Because they behave well under localization and change of coordinate, these spaces can be transferred to a manifold with boundary. For $\Omega$ a compact manifold with boundary we let $H_{(m, s)}(\Omega), \dot{H}_{(m, s)}(\Omega)$ denote the corresponding function spaces. Suppose that $(V, \varphi)$ is either a boundary or interior coordinate chart, and $\psi \in \mathscr{C}_{c}^{\infty}(V)$. A distribution $u$, defined on $\Omega$, belongs to one of these spaces if $(\psi u) \circ \varphi^{-1}$ belongs to the corresponding space in $\mathbb{R}_{+}^{n}$. Using the tubular neighborhood theorem, Theorem 6 extends to this situation:

Theorem 7 Let $\Omega$ be a compact manifold with boundary, $r$ a defining function for $b \Omega, \stackrel{\circ}{\Omega}=\{r>0\}$. If $m$ is a positive integer and $0 \leq j<m$, then the map

$$
\mathscr{C}^{\infty}(\Omega) \ni u \rightarrow \partial_{v}^{j} u(\cdot, r),
$$

for $r \geq 0$, extends as a continuous map from $H_{(m, s)}(\Omega) \rightarrow H^{s+m-j-\frac{1}{2}}(b \Omega)$. Moreover, $r \rightarrow \partial_{\nu}^{j} u(\cdot, r)$ is continuous from $[0,1)$ to $H^{s+m-j-\frac{1}{2}}(b \Omega)$. 
The connection with the analysis of boundary value problems for differential operators is provided by the following weak, but extremely useful regularity theorem. In the situation described in Theorem 7, a differential operator, $P$ of degree $m$ is called transversely elliptic if the principal symbol $\sigma_{0}(P)(x, d r)$ is invertible for all $x \in b \Omega$. In other words, the boundary of $\Omega$ is non-characteristic for $P$.

Theorem 8 Suppose that $\Omega$ is a compact manifold with boundary and $P$ is a transversely elliptic operator or order $m$. If $u \in L^{2}(\Omega)=H_{(0,0)}(\Omega)$, and $P u \in L^{2}(\Omega)$, then $u \in H_{(m,-m)}(\Omega)$.

As suggested by the identification, $L^{2}(\Omega)=H_{(0,0)}(\Omega)$ we interpret $u$ as an extendible distribution when defining $P u$. The theorem has a very useful corollary, which is a generalization of Theorem 1.

Corollary 2 If $u$, and Pu both belong to $L^{2}(\Omega)$, then, for $0 \leq j<m$ the maps $r \mapsto \partial_{\nu}^{j} u(r, \cdot)$ are continuous from $[0,1)$ to $H^{-\left(\frac{1}{2}+j\right)}(b \Omega)$. In particular,

$$
\Gamma u=\left(u(r, \cdot), \partial_{v} u(r, \cdot), \ldots, \partial_{v}^{m-1} u(r, \cdot)\right) \uparrow_{r=0}
$$

is well defined as a vector valued distribution on the boundary.

The range of $\Gamma$ consists of distributional sections of a vector bundle $E \rightarrow b \Omega$. Suppose that $\mathscr{R}$ is a pseudodifferential operator defined on $b \Omega$, which acts on sections of $E$. We define an unbounded operator $(P, \mathscr{R})$ on $L^{2}(\Omega), u \mapsto P u$, with domain

$$
\operatorname{Dom}(P, \mathscr{R})=\left\{u \in L^{2}: P u \in L^{2} \text { and } \mathscr{R} \Gamma u=0\right\} .
$$

It is not difficult to show that these operators are closed. The question of principal interest is to know when these operators are Fredholm.

Good references for the material in this section are [13], [22], and [18].

\section{Estimates for Operators Satisfying the Transmission Condition}

In the sequel we let $\Omega$ be a compact manifold with boundary, $\widetilde{\Omega}$, its double and $E, F$ complex vector bundles over $\widetilde{\Omega}$. We suppose that $P$ is a first order elliptic, differential operator from sections of $E$ to sections of $F$. In general we are rather sloppy about which bundle is which, largely leaving them out of the notation, except when absolutely necessary.

The ellipticity of $P$ means that for each non-zero $\xi \in T_{x}^{*} \widetilde{\Omega}$, the principal symbol, $p_{0}(x, \xi)$ is an invertible element of $\operatorname{Hom}\left(E_{x}, F_{x}\right)$. This implies that there is a parametrix for $P$, that is an operator $Q \in \Psi^{-1}(\widetilde{\Omega} ; F, E)$ so that

$$
P Q=\operatorname{Id}_{F}-K_{1} \quad Q P=\operatorname{Id}_{E}-K_{2}
$$

with $K_{1}, K_{2}$ smoothing operators of finite rank. (The smoothing operators are those with Schwartz kernels in $\mathscr{C}^{\infty}(\widetilde{\Omega} \times \widetilde{\Omega})$ tensored with the appropriate vector bundle.) The symbol of the operator $Q$ has an asymptotic expansion:

$$
\sigma(Q) \sim \sum_{j \geq 0} q_{j}
$$

For each $x, q_{0}(x, \xi)=p_{0}(x, \xi)^{-1}$; more generally $q_{j}(x, \xi)$ is a rational function of $\xi$ of degree $-1-j$. Indeed, the denominator of $q_{j}$ is just a power of det $p_{0}(x, \xi)$. This implies that $Q$ is an operator satisfying the following condition. 
Definition 3 A classical pseudodifferential operator in $Q \in \Psi^{*}(\widetilde{\Omega})$ satisfies the transmission condition, if, whenever $u \in \mathscr{C}^{\infty}(\bar{\Omega})$ and we denote by $u_{0}$ the extension of $u$, by zero, to all of $\widetilde{\Omega}$, then $Q u_{0} \uparrow_{\text {int } \Omega}$ extends to define an element of $\mathscr{C}^{\infty}(\bar{\Omega})$.

There is a simple symbolic criterion for a classical pseudodifferential operator to satisfy the transmission condition. It is a local condition; we introduce coordinates, $x=$ $\left(x^{\prime}, x_{n}\right)$ in a neighborhood, $U$ of a point $p \in b Y$ so that $p \leftrightarrow x=0, U \cap b Y=\left\{x_{n}=0\right\}$ and $x_{n}>0$ in the interior of $Y \cap U$. Assume that $Q$ is a classical pseudodifferential operator of order $m$ such that the (complete) symbol of $Q$ has an asymptotic expansion:

$$
\sigma(Q)(x, \xi)=q(x, \xi) \sim \sum_{j=0}^{\infty} q_{j}(x, \xi),
$$

where

$$
q_{j}(x, \lambda \xi)=\lambda^{m-j} q_{j}(x, \xi) \text { for } \lambda>0 .
$$

The operator satisfies the transmission condition with respect to $Y$, provided

$$
q_{j}\left(x^{\prime}, x_{n}, \xi^{\prime}, \xi_{n}\right)-e^{-\pi i(m+j)} q_{j}\left(x^{\prime}, x_{n},-\xi^{\prime},-\xi_{n}\right) \text { for } j=0,1, \ldots,
$$

vanish to infinite order along the inward pointing co-normal bundle to $b Y$, i.e., where $x_{n}=0, \xi^{\prime}=0$ and $\xi_{n}>0$. As shown in [14], this is a coordinate invariant condition and so can be used to check the transmission condition for pseudodifferential operators defined on manifolds.

In our applications the terms in the asymptotic expansion of $\sigma(Q)$ are homogeneous, rational functions of $\xi$, which therefore satisfy the following condition:

$$
q_{j}(x, \lambda \xi)=\lambda^{m-j} q_{j}(x, \xi), \text { for all } \lambda \in \mathbb{C}^{*} .
$$

We call these properties the strengthened transmission condition. In the arguments that follow we often use this stronger condition as it simplifies the exposition.

\section{$\Gamma_{+}$}

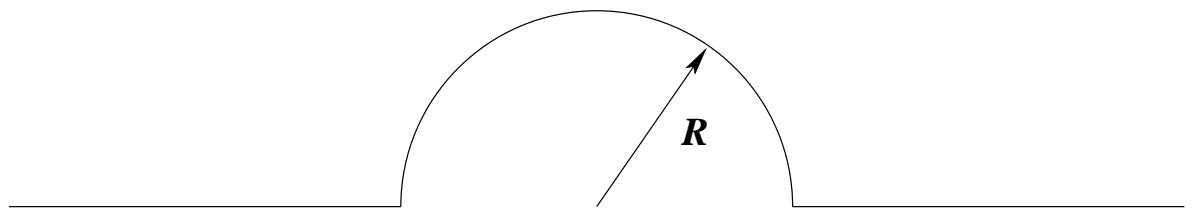

Figure 1 The integration contour.

To understand the analytic properties underlying the transmission condition we consider a function $u \in \mathscr{C}_{c}^{\infty}\left(\overline{\mathbb{R}}_{+}^{n}\right)$. If

$$
a\left(x^{\prime}, \xi_{n}\right)=\int_{0}^{\infty} u\left(x^{\prime}, x_{n}\right) e^{-i x_{n} \xi_{n}} d x_{n},
$$

then $a\left(x^{\prime}, \xi_{n}\right)$ has an asymptotic expansion

$$
a\left(x^{\prime}, \xi_{n}\right) \sim \sum_{j=1}^{\infty} \frac{\partial_{x_{n}}^{j} u\left(x^{\prime}, 0\right)}{\left(i \xi_{n}\right)^{j}}=\sum_{j=1}^{\infty} a_{j}\left(x^{\prime}, \xi_{n}\right) .
$$


Let $\Gamma^{+} \subset \mathbb{C}$ be the contour $(-\infty, R] \cup\left\{R e^{i \theta}: \theta \in[\pi, 0]\right\} \cup[R, \infty)$, see Figure 1 . The function $a_{j}$ satisfies

$$
a_{j}\left(x^{\prime}, \xi_{n}\right)=a_{j}\left(x^{\prime}, 1\right) \xi_{n}^{-j}
$$

For such a function, the oscillatory integral

$$
\int_{\Gamma^{+}} a_{j}\left(x^{\prime}, \xi_{n}\right) e^{i x_{n} \xi_{n}} d \xi_{n}
$$

is well defined. In fact, if $x_{n}>0$, then a simple contour deformation argument shows that this integral vanishes. As an oscillatory integral, this remains true for a function of the form $a\left(x^{\prime}\right) \xi_{n}^{j}$, for any $j \in \mathbb{Z}$. Indeed, for $x_{n}>0$, and $l \in \mathbb{N}$, as an oscillatory integral,

$$
\int_{\Gamma_{+}} \xi_{n}^{j} e^{i x_{n} \xi_{n}} d \xi_{n}=\left[\partial_{x_{n}}\right]^{l} \int_{\Gamma_{+}} \xi_{n}^{j}\left(i \xi_{n}\right)^{-l} e^{i x_{n} \xi_{n}} d \xi_{n} .
$$

Choosing $l>j+1$, it follows easily that the right hand side is zero, for $x_{n}>0$.

Now suppose that $v$ is a compactly supported distribution with a representation, as an oscillatory integral, of the form:

$$
v\left(x^{\prime}, x_{n}\right)=\frac{1}{2 \pi} \int_{-\infty}^{\infty} b\left(x^{\prime}, \xi_{n}\right) e^{i x_{n} \xi_{n}} d \xi_{n},
$$

where $b$ is a classical symbol having an asymptotic expansion

$$
b\left(x^{\prime}, \xi_{n}\right) \sim \sum_{j=0}^{\infty} b_{j}\left(x^{\prime}, \xi_{n}\right)
$$

with $b_{j}\left(x^{\prime}, \xi_{n}\right)=b_{j}\left(x^{\prime}, 1\right) \xi_{n}^{m-j}$, for all $j$. As $b$ is a symbol, the functions $\left\{b_{j}\left(x^{\prime}, 1\right)\right\}$ are in $\mathscr{C}^{\infty}\left(\mathbb{R}^{n-1}\right)$. For any $N>0$, and $x_{n}>0$ we observe that

$$
\begin{aligned}
v\left(x^{\prime}, x_{n}\right) & =v\left(x^{\prime}, x_{n}\right)-\frac{1}{2 \pi} \sum_{j=0}^{N} \int_{\Gamma^{+}} b_{j}\left(x^{\prime}, \xi_{n}\right) e^{i x_{n} \xi_{n}} d \xi_{n} \\
& =\frac{1}{2 \pi}\left[\int_{\left|\xi_{n}\right|>R}\left[b\left(x^{\prime}, \xi_{n}\right)-\sum_{j=0}^{N} b_{j}\left(x^{\prime}, \xi_{n}\right)\right] e^{i x_{n} \xi_{n}} d \xi_{n}+\right. \\
& \left.\int_{-R}^{R} b\left(x^{\prime}, \xi_{n}\right) e^{i x_{n} \xi_{n}} d \xi_{n}-\int_{\pi}^{0} \sum_{j=0}^{N} b_{j}\left(x^{\prime}, R e^{i \theta}\right) e^{i x_{n} R e^{i \theta}} R d e^{i \theta}\right]
\end{aligned}
$$

The integrals over compactly sets define smooth functions in $\overline{\mathbb{R}}_{+}^{n}$, and the integral over $\left|\xi_{n}\right|>R$ is a $C^{N-|m|-1}\left(\overline{\mathbb{R}}_{+}^{n}\right)$ function. As $N$ is arbitrary, the restriction of $v$ to int $\mathbb{R}_{+}^{n}$ extends to define an element of $\mathscr{C}^{\infty}\left(\overline{\mathbb{R}}_{+}^{n}\right)$. This simple analytic continuation argument explains the essence of the transmission condition. In this section we use this sort of contour deformation to establish mapping properties for $Q$ acting on $H^{s}(\Omega)$ as well as its effect on distributions supported on $b \Omega$ itself. The result we obtain is:

Theorem 9 Suppose that $Q$ is a classical pseudodifferential operator of order $m$, on $\widetilde{\Omega}$, satisfying the strengthened transmission condition with respect to $\Omega$. For $s \geq 0$, $Q: H^{s}(\Omega) \rightarrow H^{s-m}(\Omega)$. 
To prove this theorem we use the following local result.

Proposition 2 Let $Q$ be an classical pseudodifferential operator of integral degree $m$ on $\mathbb{R}^{n}$ satisfying the strengthened transmission condition with respect to $\mathbb{R}_{+}^{n}$. If $s \geq 0$, and $f \in H_{\text {comp }}^{s}\left(\mathbb{R}_{-}^{n}\right)$, then, for any $k \in \mathbb{N}_{0}$, we have:

$$
\text { Qf } \uparrow_{\mathbb{R}_{+}^{n}} \in H_{(k, s-m-k), l o c}\left(\mathbb{R}_{+}^{n}\right)
$$

Proof Because pseudodifferential operators are pseudolocal, it follows that the restriction $Q f\left\lceil_{\text {int }} \mathbb{R}_{+}^{n}\right.$ is smooth. As $\mathscr{C}^{\infty}\left(\overline{\mathbb{R}}_{-}^{n}\right)$ is dense in $H^{s}\left(\mathbb{R}_{-}^{n}\right)$, it suffices to show that, for every $s$, and $k$, there is a constant $C_{s, k}$, such that for $f \in \mathscr{C}^{\infty}\left(\overline{\mathbb{R}}_{-}^{n}\right)$, and $\varphi \in \mathscr{C}_{c}^{\infty}\left(\overline{\mathbb{R}}_{+}^{n}\right)$, we have

$$
\|\varphi Q(f)\|_{(k, s-m-k)} \leq C_{s, k}\|f\|_{H^{s}\left(\mathbb{R}_{-}^{n}\right)} .
$$

Let $q \sim \sum q_{j}$, where $q_{j}(x, \xi)$, is a homogeneous rational function in $\xi$, of degree $m-j$.

Remark 2 The following argument is modeled on the proof of Theorem 18.2.17 in [14]. Let $\phi \in \mathscr{C}_{c}^{\infty}(\mathbb{R})$, with support in $[-1,1]$, and total integral 1. For each $\epsilon>0$, we let $\phi_{\epsilon}(x)=\epsilon^{-1} \phi\left(\epsilon^{-1} x\right)$. To be entirely rigorous in the derivation of the formulæ below, we should first work with the regularized functions $f_{\epsilon}=f *_{x_{n}} \phi_{\epsilon}$, and then allow $\epsilon$ to tend to zero. For $\epsilon>0$ these functions belong to $\mathscr{C}_{c}^{\infty}\left(\mathbb{R}^{n}\right)$. To highlight the important details, we proceed somewhat formally, working directly with $f \in \mathscr{C}_{c}^{\infty}\left(\overline{\mathbb{R}}_{-}^{n}\right)$. The more precise argument is quite standard, and can be found in see [14].

We begin with a lemma. Let $\psi\left(\xi^{\prime}\right)$ be a smooth function, with $\psi\left(\xi^{\prime}\right)=0$, if $\left\|\xi^{\prime}\right\|<1$, and $\psi\left(\xi^{\prime}\right)=1$, for $\left\|\xi^{\prime}\right\|>2$.

Lemma 2 If $s \in \mathbb{R}$ and $f \in H_{\text {comp }}^{s}\left(\mathbb{R}_{-}^{n}\right)$, then

$$
Q_{0}(f)=\left[\frac{1}{(2 \pi)^{n}} \int_{\mathbb{R}^{n}} q(x, \xi)\left(1-\psi\left(\xi^{\prime}\right)\right) \hat{f}(\xi) e^{i x \cdot \xi} d \xi\right]_{\mathbb{R}_{+}^{n}}
$$

belongs to $\mathscr{C}^{\infty}\left(\overline{\mathbb{R}}_{+}^{n}\right)$.

Proof of the Lemma For each $N$, there is an $R$ so that, if $\left\|\xi^{\prime}\right\| \leq 2$, then the poles of $\left\{q_{j}\left(x, \xi^{\prime}, \xi_{n}\right): j=0, \ldots, N\right\}$ lie inside $D_{R}(0)$. Because $f$ is supported in the lower half space, its Fourier transform extends to be a holomorphic function of $\xi_{n}$ in the upper half space. Let $C_{R}^{+}$denote the arc, in the $\xi_{n}$-plane, $\left\{\xi_{n}=R e^{i \theta}, \theta \in[\pi, 0]\right\}$. Using the analyticity properties of $\hat{f}$ and the $q_{j}$, we can therefore argue as in equation (86), that for $x_{n}>0$, we have

$$
\begin{aligned}
Q_{0}(f)\left(x^{\prime}, x_{n}\right) & =\frac{1}{(2 \pi)^{n}} \int_{\mathbb{R}^{n-1}} \int_{\left|\xi_{n}\right|>R}\left(q(x, \xi)-\sum_{j=0}^{N} q_{j}(x, \xi)\right) e^{i x_{n} \xi_{n}}\left(1-\psi\left(\xi^{\prime}\right)\right) \times \\
& \hat{f}(\xi) d \xi_{n} e^{i x \cdot \xi^{\prime}} d \xi^{\prime} \\
& +\frac{1}{(2 \pi)^{n}} \int_{\mathbb{R}^{n-1}} \int_{\left|\xi_{n}\right| \leq R} q(x, \xi) e^{i x_{n} \xi_{n}}\left(1-\psi\left(\xi^{\prime}\right)\right) \hat{f}(\xi) d \xi_{n} e^{i x \cdot \xi^{\prime}} d \xi^{\prime} \\
& -\frac{1}{(2 \pi)^{n}} \int_{\mathbb{R}^{n-1}} \int_{C_{R}^{+}} \sum_{j=0}^{N} q_{j}(x, \xi) e^{i x_{n} \xi_{n}}\left(1-\psi\left(\xi^{\prime}\right)\right) \hat{f}(\xi) d \xi_{n} e^{i x \cdot \xi^{\prime}} d \xi^{\prime}
\end{aligned}
$$


By taking $N$ large, we can make the difference appearing in the first integral vanish, when $\|\xi\| \rightarrow \infty$, as rapidly as we like, thereby making the first integral as smooth as we wish. The other two terms are integrals over compact sets, which therefore define $\mathscr{C}^{\infty}$-functions in $\left\{x_{n} \geq 0\right\}$. The existence of an estimate, as above, follows from the closed graph theorem.

From the lemma it suffices to consider

$$
Q_{1}(f)(x)=\left[\frac{1}{(2 \pi)^{n}} \int_{\mathbb{R}^{n}} q(x, \xi) \psi\left(\xi^{\prime}\right) \hat{f}(\xi) e^{i x \cdot \xi} d \xi\right]_{\mathbb{R}_{+}^{n}}
$$

for $f \in \mathscr{C}_{c}^{\infty}\left(\overline{\mathbb{R}}_{-}^{n}\right)$. For each $j \in \mathbb{N}_{0}$, define the pseudodifferential operator:

$$
Q_{j 1}(f)(x)=\left[\frac{1}{(2 \pi)^{n}} \int_{\mathbb{R}^{n}} q_{j}(x, \xi) \psi\left(\xi^{\prime}\right) \hat{f}(\xi) e^{i x \cdot \xi} d \xi\right]_{\mathbb{R}_{+}^{n}}
$$

For $N \in \mathbb{N}$, the difference $Q-\sum_{j<N} Q_{j 1}$ is a pseudodifferential operator of order $-N$, and therefore it suffices to prove estimates for $Q_{j 1}(f), j=0, \ldots$

To prove these estimates, we take the tangential Fourier transform of $Q_{j 1}(f)$. We let

$$
\widetilde{q}_{j}\left(\eta^{\prime}, x_{n}, \xi\right)=\int_{\mathbb{R}^{n-1}} q_{j}\left(x^{\prime}, x_{n}, \xi\right) e^{-i x^{\prime} \cdot \eta^{\prime}} d x^{\prime} .
$$

From the symbolic estimates, it follows that, for each $M \in \mathbb{N}$, there is a constant, $C_{M}$, so that

$$
\widetilde{q}_{j}\left(\eta^{\prime}, x_{n}, \xi\right) \leq C_{M} \frac{\|\xi\|^{m-j}}{\left(1+\left\|\eta^{\prime}\right\|\right)^{M}} .
$$

For $s \geq 0$, there is a universal constant, $C_{s}^{\prime}$ so that if $f \in H^{s}\left(\mathbb{R}_{-}^{n}\right)$, then

$$
\int_{\mathbb{R}^{n-1}} \int_{-\infty}^{0}\left|\tilde{f}\left(\xi^{\prime}, x_{n}\right)\right|^{2}\left(1+\left\|\xi^{\prime}\right\|\right)^{2 s} d x_{n} d \xi^{\prime} \leq C_{s}^{\prime}\|f\|_{H^{s}\left(\mathbb{R}_{-}^{n}\right)}
$$

Moreover, $\hat{f}\left(\xi^{\prime}, \xi_{n}\right)$ analytically extends to $\left\{\operatorname{Im} \xi_{n}>0\right\}$; for $\beta>0$, the Cauchy-Schwarz inequality implies the estimate:

$$
\begin{aligned}
\left|\hat{f}\left(\xi^{\prime}, \alpha+i \beta\right)\right|^{2} & =\left|\int_{-\infty}^{0} \tilde{f}\left(\xi^{\prime}, x_{n}\right) e^{-i x_{n}(\alpha+i \beta)} d x_{n}\right|^{2} \\
& \leq \frac{\int_{-\infty}^{0}\left|\tilde{f}\left(\xi^{\prime}, x_{n}\right)\right|^{2} d x_{n}}{2 \beta}
\end{aligned}
$$

As $q_{j}\left(x, \xi^{\prime}, \xi_{n}\right)$ is homogeneous in $\xi$, its poles, as a function of $\xi_{n}$, in the upper half plane, are of the form $\left\{\left\|\xi^{\prime}\right\| w_{l}\left(\omega^{\prime}\right): j=1, \ldots, L\right\}$; we let

$$
w_{l}\left(\omega^{\prime}\right)=\alpha_{l}\left(\omega^{\prime}\right)+i \beta_{l}\left(\omega^{\prime}\right) .
$$


Here $\left\|\xi^{\prime}\right\| \omega^{\prime}=\xi^{\prime}$. We can use contour integration to evaluate the $\xi_{n}$-integral. Assuming, for the moment, that all the poles of $q_{j}$ are simple, we obtain that

$$
\begin{aligned}
Q_{j 1}(f)\left(x^{\prime}, x_{n}\right)= & \sum_{l=1}^{L} \frac{i}{(2 \pi)^{n-1}} \int_{\mathbb{R}^{n-1}} q_{j}^{(l)}\left(x^{\prime}, x_{n}, \xi^{\prime},\left\|\xi^{\prime}\right\| w_{l}\left(\omega^{\prime}\right)\right) \psi\left(\xi^{\prime}\right) \\
& \hat{f}\left(\xi^{\prime},\left\|\xi^{\prime}\right\| w_{l}\left(\omega^{\prime}\right)\right) e^{i x_{n}\left\|\xi^{\prime}\right\| w_{l}\left(\omega^{\prime}\right)} e^{i x^{\prime} \cdot \xi^{\prime}} d \xi^{\prime},
\end{aligned}
$$

where

$$
q_{j}^{(l)}\left(x, \xi^{\prime}, \xi_{n}\right)=\left(\xi_{n}-\left\|\xi^{\prime}\right\| w_{l}\left(\omega^{\prime}\right)\right) q_{j}\left(x, \xi^{\prime}, \xi_{n}\right) .
$$

Away from $\xi^{\prime}=0$, these are homogeneous symbols of degree $m-j+1$. Clearly it suffices to separately estimate each term in (98). For each $M$, there is a constant $C_{M}$ such that the tangential Fourier transform of $q_{j}^{(l)}$ satisfies the estimate:

$$
\widetilde{q}_{j}^{(l)}\left(\eta^{\prime}, x_{n}, \xi\right) \leq C_{M} \frac{\|\xi\|^{m-j+1}}{\left(1+\left\|\eta^{\prime}\right\|\right)^{M}} .
$$

This shows that the tangential Fourier transform of each term in the sum satisfies the estimate:

$$
\left|\widetilde{Q}_{j 1}^{(l)} f\left(\eta^{\prime}, x_{n}\right)\right| \leq C \int_{\mathbb{R}^{n-1}} \frac{\left\|\xi^{\prime}\right\|^{m-j+1} \psi\left(\xi^{\prime}\right)\left|\hat{f}\left(\xi^{\prime},\left\|\xi^{\prime}\right\| w_{l}\left(\omega^{\prime}\right)\right)\right| e^{-x_{n} \beta_{l}\left(\omega^{\prime}\right)\left\|\xi^{\prime}\right\|} d \xi^{\prime}}{\left(1+\left\|\xi^{\prime}-\eta^{\prime}\right\|\right)^{M}}
$$

We apply the Cauchy-Schwarz inequality to the right hand side of (101) to obtain:

$$
\begin{aligned}
\left|\widetilde{Q}_{j 1}^{(l)} f\left(\eta^{\prime}, x_{n}\right)\right|^{2} \leq & C \int_{\mathbb{R}^{n-1}} \frac{\left\|\xi^{\prime}\right\|^{2(m-j+1)} \psi\left(\xi^{\prime}\right)\left|\hat{f}\left(\xi^{\prime},\left\|\xi^{\prime}\right\| w_{l}\left(\omega^{\prime}\right)\right)\right|^{2} e^{-2 x_{n} \beta_{l}\left(\omega^{\prime}\right)\left\|\xi^{\prime}\right\|} d \xi^{\prime}}{\left(1+\left\|\xi^{\prime}-\eta^{\prime}\right\|\right)^{M}} \times \\
& \int_{\mathbb{R}^{n-1}} \frac{d \xi^{\prime}}{\left(1+\left\|\xi^{\prime}-\eta^{\prime}\right\|\right)^{M}}
\end{aligned}
$$

For $M$ sufficiently large, the second integral in (102) converges. By ellipticity and compactness, the imaginary part of the exponent $\beta_{l}\left(\omega^{\prime}\right) \geq \beta>0$, as $\omega^{\prime}$ varies over the unit sphere. Using this estimate, and the estimate in (96) we see that

$$
\begin{aligned}
& \int_{\mathbb{R}^{n-1}} \int_{0}^{\infty}\left|\widetilde{Q}_{j 1}^{(l)} f\left(\eta^{\prime}, x_{n}\right)\right|^{2}\left(1+\left\|\eta^{\prime}\right\|\right)^{2(s+j-m)} d x_{n} d \eta^{\prime} \leq \\
& \int_{\mathbb{R}^{n-1}} \int_{\mathbb{R}^{n-1}} \frac{\psi\left(\xi^{\prime}\right)\left\|\xi^{\prime}\right\|^{2(m-j+1)}\left(1+\left\|\eta^{\prime}\right\|\right)^{2(s+j-m)}}{\left(1+\left\|\xi^{\prime}-\eta^{\prime}\right\|\right)^{M} \beta^{2}\left\|\xi^{\prime}\right\|^{2}} \times \int_{-\infty}^{0}\left|\tilde{f}\left(\xi^{\prime}, y_{n}\right)\right|^{2} d y_{n} d \xi^{\prime} d \eta^{\prime} .
\end{aligned}
$$

One power of $\left\|\xi^{\prime}\right\|$ in the denominator results from performing the $x_{n}$-integral, and the other comes from (96). To complete the proof we use the following elementary lemma:

Lemma 3 If $t \in \mathbb{R}$ and $M>2 t+n$, then there is a constant $C$ so that:

$$
\int_{\mathbb{R}^{n-1}} \frac{\left(1+\left\|\eta^{\prime}\right\|\right)^{2 t}}{\left(1+\left\|\xi^{\prime}-\eta^{\prime}\right\|\right)^{M}} \leq C\left(1+\left\|\xi^{\prime}\right\|\right)^{2 t}
$$


The proof is left to the reader.

Interchanging the order of the $\eta^{\prime}$ and $\xi^{\prime}$ integrations in (103), we apply the lemma to obtain that

$$
\begin{aligned}
\int_{\mathbb{R}^{n-1}} \int_{0}^{\infty}\left|\widetilde{Q}_{j 1}^{(l)} f\left(\eta^{\prime}, x_{n}\right)\right|^{2}\left(1+\left\|\eta^{\prime}\right\|\right)^{2(s+j-m)} d x_{n} d \eta^{\prime} \leq \\
C \int_{\mathbb{R}^{n-1}}\left(1+\left\|\xi^{\prime}\right\|\right)^{2 s} \int_{-\infty}^{0}\left|\tilde{f}\left(\xi^{\prime}, y_{n}\right)\right|^{2} d y_{n} d \xi^{\prime} .
\end{aligned}
$$

In light of equation (95), this proves the proposition, for $k=0$, under the assumption that all poles of $q_{j}$ are simple. The latter assumption is easily removed, by using Cauchy's formula

$$
\frac{k !}{2 \pi i} \int_{\mathbb{R}} \frac{f(w) d w}{(z-w)^{k+1}}=\partial_{z}^{k} f(z),
$$

the Leibniz formula, and symbolic estimates. It is seen to give the same result, as in the simple case, if we replace (96) with the estimate

$$
\left|\partial_{\xi_{n}}^{k} \hat{f}\left(\xi^{\prime}, \alpha+i \beta\right)\right|^{2} \leq C_{k} \frac{\int_{-\infty}^{0}\left|\tilde{f}\left(\xi^{\prime}, x_{n}\right)\right|^{2} d x_{n}}{\beta^{2 k+1}}
$$

To estimate derivatives in the $x_{n}$ direction, we simply differentiate (98). Each derivative replaces the symbol, in $\xi^{\prime}$, with a symbol of one higher degree and the argument is otherwise the same.

Proof of the Theorem Let $f \in H^{s}(\Omega)$. Using the Seeley extension theorem we know that there is a constant $C_{s}$, and an extension $f^{\prime}$ of $f$ to $\widetilde{\Omega}$, so that

$$
\left\|f^{\prime}\right\|_{H^{s}(\widetilde{\Omega})} \leq C_{s}\|f\|_{H^{s}(\Omega)} .
$$

Because $Q$ is a pseudodifferential operator of order $m$, it follows that there is a constant $C_{S}^{\prime}$ so that

$$
\left\|Q f^{\prime}\right\|_{H^{s-m}(\widetilde{\Omega})} \leq C_{s}^{\prime}\left\|f^{\prime}\right\|_{H^{s}(\widetilde{\Omega})} .
$$

In light of the definition of the norm on $H^{s}(\Omega)$, this shows that $Q f^{\prime}\left\lceil\Omega \in H^{s-m}(\Omega)\right.$. If we let

$$
f_{-}=\left\{\begin{array}{l}
f^{\prime}\lceil\widetilde{\Omega} \backslash \Omega \\
0 \text { in } \Omega,
\end{array}\right.
$$

then we need only show that $Q f_{-} \Gamma_{\Omega} \in H^{s-m}(\Omega)$. To prove this we observe that it is enough to prove estimates in boundary coordinate charts.

$$
\text { If } s-m \leq 0 \text {, then } H_{(0, s-m)}(\Omega) \subset H^{s-m}(\Omega) \text {. If } s-m>0 \text {, then }
$$

The needed estimates follow immediately from the proposition, and these inclusions, thus completing the proof of the Theorem. 
Remark 3 The results in Theorem 10 can easily be extended, by duality to $s \geq$ $-\frac{1}{2}$. The strengthened transmission condition is invariant under transposition, so $Q^{t}$ also satisfies it. The facts that $\left[H^{s}(\Omega)\right]^{\prime}=\dot{H}^{-s}(\Omega)$ and $H^{s}(\Omega) \simeq \dot{H}^{s}(\Omega)$ for $s \in\left[-\frac{1}{2}, \frac{1}{2}\right]$, therefore allow us to extend the theorem to $s \geq-\frac{1}{2}$.

Using essentially the same argument we can treat the case of a single layer potential:

Theorem 10 Suppose that $Q$ is a classical pseudodifferential operator of order $m$, on $\widetilde{\Omega}$, satisfying the strengthened transmission condition with respect to $\Omega$. If $r$ is a defining function for $b \Omega$, and $f \in \mathscr{C}^{\infty}(b \Omega)$, then $Q(f \otimes \delta(r))$ extends to define a function in $\mathscr{C}^{\infty}(\bar{\Omega})$. If $s \geq-\frac{1}{2}$ and $f \in H^{s}(b \Omega)$, then, for $k \in \mathbb{N}_{0}$, we have that $Q(f \otimes \delta(r)) \in$ $H_{\left(k, s-m-k-\frac{1}{2}\right)}(\Omega)$.

Remark 4 Similar results hold for multiple layer potentials, i.e. distributions of the form $Q\left(f \otimes \delta^{[j]}(r)\right)$. Detailed proofs of these results, in somewhat greater generality are given in [14].

\section{The Calderon Projection}

We now let $Q$ denote a parametrix for a first order differential operator, $P$ acting between sections of a vector bundles $E$, and $F$ :

$$
P: \mathscr{C}^{\infty}(\widetilde{\Omega} ; E) \longrightarrow \mathscr{C}^{\infty}(\widetilde{\Omega} ; F),
$$

A typical example is a Dirac operator.

Example 1 On a $n$-dimensional, complex Kähler manifold, $X$, the bundle of complex spinors is isomorphic to the direct sum of the $(0, q)$-forms:

$$
\$ \simeq \bigoplus_{q=0}^{n} \Lambda^{0, q} X .
$$

In this case the Dirac operator, $\partial$ is nothing but $\bar{\partial}+\bar{\partial}^{*}$. This is evidently a self adjoint operator. Notice that the collections of even and odd degree forms define subbundles of $\$$ :

$$
\oiint^{\mathrm{e}}=\bigoplus_{q=0}^{n / 2} \Lambda^{0,2 q} X \quad \oint^{\mathrm{o}}=\bigoplus_{q=0}^{n / 2} \Lambda^{0,2 q+1} X ;
$$

these are often called the bundles of even and odd spinors. The Dirac operator then maps sections of $\not^{\mathrm{e}}$ to $\$^{\circ}$, and vice versa:

$$
\mathrm{\partial}^{\mathrm{eo}}: \mathscr{C}^{\infty}\left(X ; \not^{\mathrm{eo}}\right) \longrightarrow \mathscr{C}^{\infty}\left(X ; \not^{\mathrm{oe}}\right) .
$$

The operators $\partial^{\text {eo }}$ are called the chiral Dirac operators. At least formally $\left[\check{\partial}^{\mathrm{eo}}\right]^{*}=\check{\partial}^{\mathrm{oe}}$. Indeed, if $X$ is compact, then ${ }^{\mathrm{eeo}}$ are Fredholm operators with

$$
\operatorname{Ind}\left(\check{\partial}^{\mathrm{e}}\right)=\operatorname{dim} \operatorname{ker} \check{\partial}^{\mathrm{e}}-\operatorname{dim} \operatorname{ker} ð^{\mathrm{o}}=\sum_{j=0}^{n} \operatorname{dim} H^{0, q}(X)(-1)^{q} .
$$

To simplify the discussion a little bit, we assume that $P$ is actually invertible, so that $Q$ can be taken to be a fundamental solution; that is the error terms in (73) actually vanish. For the case of a Dirac operator this can always be arranged. 
The operator $Q$ is a classical pseudodifferential operator of order -1 . Indeed, its symbol has an asymptotic expansion:

$$
\sigma(Q)(x, \xi) \sim \sum_{j=0}^{\infty} q_{j}(x, \xi),
$$

with $q_{j}(x, \xi)$ a rational functional of $\xi$, homogeneous of degree $-1-j$. The denominator of $q_{j}$ is a power of $\operatorname{det}\left(p_{0}(x, \xi)\right)$.

We suppose that a Riemannian metric is fixed on $\widetilde{\Omega}$, and Hermitian inner products on $E, F$, though this data is often suppressed in what follows. When needed $\langle\cdot, \cdot\rangle_{E}$, e.g. denotes the fiber inner product on $E$. If $\mathscr{H}$ is a Hilbert space, then $\langle\cdot, \cdot\rangle_{\mathscr{H}}$ denotes the Hilbert space inner product. Fix a defining function $r$ for $b \Omega$ in $\widetilde{\Omega}$, such that $d r$ has unit length along $b \Omega$.

We let $\Omega_{+}$denote the subset of $\widetilde{\Omega}$ where $r \geq 0$, and $\Omega_{-}$the subset where $r \leq 0$. We also let $Y_{\epsilon}$ denote the hypersurface $\{r=\epsilon\}$. As $Q$ is a fundamental solution, it is clear that $u=Q(g \otimes \delta(r))$ belongs to the nullspace of $P$ on $\widetilde{\Omega} \backslash b \Omega$. We denote the restrictions to the components of the complement of $b \Omega$ by $u_{ \pm}$. It follows from Theorem 10 that if $g \in H^{s}\left(b \Omega ; F \uparrow_{b \Omega}\right)$, then $u_{ \pm} \in H_{\left(1, s-\frac{1}{2}\right)}\left(\Omega_{ \pm} ; E\right)$. Let $\tau_{\epsilon}$ denote restriction to $\{r=\epsilon\}$. From Theorem 7 it follows that $\tau_{\epsilon} u$ is well defined as an element of $H^{s}\left(Y_{\epsilon}\right)$, moreover the maps

$$
\begin{aligned}
& {[0,1] \ni \epsilon \mapsto \tau_{\epsilon} u_{+}} \\
& {[-1,0] \ni \epsilon \mapsto \tau_{\epsilon} u_{-}}
\end{aligned}
$$

are continuous. Note, however, that generally $\tau_{0} u_{+} \neq \tau_{0} u_{-}$.

We need to establish the properties of the maps

$$
\mathscr{P}_{ \pm} f=\lim _{\epsilon \rightarrow 0^{ \pm}} \tau_{\epsilon} Q\left(\sigma_{0}(P, \pm d r)(f \otimes \delta(r))\right) .
$$

Here $f$ is a distributional section of $E\left\lceil_{b \Omega}\right.$, and $\sigma_{0}(P, d r)$ is the principal symbol of $P$ in the co-normal direction $d r$. If $u_{ \pm}$belongs to the nullspace of $P$ on $\Omega_{ \pm}$, then it follows from Green's formula, and the fact that $Q$ is a fundamental solution that

$$
u_{ \pm}(p)=Q\left(\sigma_{0}(P, \pm d r)\left[u_{ \pm}\left\lceil b \Omega_{ \pm} \otimes \delta(r)\right]\right)(p) \text { for } p \in \Omega_{ \pm} .\right.
$$

Hence $\mathscr{P}_{ \pm} u_{ \pm}=u_{ \pm} \Upsilon_{b \Omega_{ \pm}}$. This shows that $\mathscr{P}_{ \pm}$are projection operators. These are the Calderon projectors for the operator $P$.

As $Q$ is a fundamental solution,

$$
P Q\left[\sigma_{0}(P, d r) f \otimes \delta(r)\right]=\sigma_{0}(P, d r) f \otimes \delta(r) .
$$

Hence, if $f$ is a smooth section of $E$ along $b \Omega$ and $\varphi$ is a smooth section of $F$ in $\widetilde{\Omega}$, then

$$
\begin{aligned}
\int_{b \Omega}\left\langle\sigma_{0}(P, d r) f, \varphi\right\rangle_{F}= & \lim _{\epsilon \rightarrow 0^{+}} \int_{\{|r|>\epsilon}\left\langle Q\left(\sigma_{0}(P, d r) f \otimes \delta(r)\right), P^{t} \varphi\right\rangle_{E} \\
& =\lim _{\epsilon \rightarrow 0^{+}}\left[\int_{\{r=\epsilon\}}\left\langle Q\left(\sigma_{0}(P, d r) f \otimes \delta(r)\right), \sigma\left(P^{t}, d r\right) \varphi\right\rangle_{E}-\right. \\
& \left.\int_{\{r=-\epsilon\}}\left\langle Q\left(\sigma_{0}(P, d r) f \otimes \delta(r)\right), \sigma\left(P^{t}, d r\right) \varphi\right\rangle_{E}\right] \\
& =\left\langle\sigma_{0}(P, d r)\left(\mathscr{P}_{+}+\mathscr{P}_{-}\right) f, \varphi\right\rangle_{L^{2}(b \Omega ; F)} .
\end{aligned}
$$


As $\varphi$ is an arbitrary smooth section of $F$ and $\sigma_{0}(P, d r)$ is invertible, we see that

$$
f=\left(\mathscr{P}_{+}+\mathscr{P}_{-}\right) f \text {. }
$$

Arguing as in the previous section we can use contour integration in the $\xi_{n}$-variable to obtain a formula for $Q(g \otimes \delta(r))$. Here $g$ is a smooth section of $F\left\lceil_{b \Omega}\right.$. As before, this is a local problem, we introduce coordinates $\left(x^{\prime}, x_{n}\right)$, in a neighborhood of $U$ of $p \in b \Omega_{+}$ so that

$$
\Omega_{ \pm} \cap U=\left\{ \pm x_{n} \geq 0\right\} .
$$

We let $\psi \in \mathscr{C}^{\infty}\left(\mathbb{R}^{n-1}\right)$ be a function that is 0 in a neighborhood of 0 and 1 outside the ball of radius 2; we can again show that, for $x_{n} \neq 0$, the functions

$$
Q(g \otimes \delta(r))-\frac{1}{(2 \pi)^{n}} \int_{\mathbb{R}^{n-1}} \int_{-\infty}^{\infty} q\left(x^{\prime}, x_{n}, \xi^{\prime}, \xi_{n}\right) \hat{g}\left(\xi^{\prime}\right) \psi\left(\xi^{\prime}\right) e^{i x_{n} \xi_{n}} d \xi_{n} e^{i x^{\prime} \cdot \xi^{\prime}} d \xi^{\prime}
$$

extend smoothly to both $\overline{\Omega_{ \pm}}$. Thus the restrictions to $\left\{x_{n}=0\right\}$ are themselves smoothing operators.

We study the symbolic properties of $\mathscr{P}_{ \pm}$by evaluating the $\xi_{n}$-integrals:

$$
\frac{1}{(2 \pi)^{n}} \int_{\mathbb{R}^{n-1}} \int_{-\infty}^{\infty} q_{j}\left(x^{\prime}, x_{n}, \xi^{\prime}, \xi_{n}\right) \hat{g}\left(\xi^{\prime}\right) \psi\left(\xi^{\prime}\right) e^{i x_{n} \xi_{n}} d \xi_{n} e^{i x^{\prime} \cdot \xi^{\prime}} d \xi^{\prime},
$$

for $x_{n} \neq 0$, by using contour integration. If $x_{n}>0$ then, for each $\xi^{\prime}$, we use a contour that includes a semi-circle in the upper half plane enclosing the poles of $q_{j}\left(x^{\prime}, x_{n}, \xi^{\prime}, \xi_{n}\right)$, whereas if $x_{n}<0$, then we use a contour in the lower half plane enclosing the poles in the lower half plane. In fact, the locations of the poles of the $q_{j}$ do not depend on $j$, but coincide with the zeros of det $p_{0}\left(x^{\prime}, x_{n}, \xi^{\prime}, \xi_{n}\right)$. Since

$$
p_{0}\left(x^{\prime}, x_{n}, \xi^{\prime}, \xi_{n}\right)=\left\|\xi^{\prime}\right\| p_{0}\left(x^{\prime}, x_{n}, \frac{\xi^{\prime}}{\left\|\xi^{\prime}\right\|}, \frac{\xi_{n}}{\left\|\xi^{\prime}\right\|}\right),
$$

the poles are also homogeneous of degree 1 in $\left\|\xi^{\prime}\right\|$. As $P$ is elliptic, $p_{0}\left(x, \omega^{\prime}, \xi_{n}\right)$ is invertible for $\xi_{n}$ on the real axis, here $\omega^{\prime}=\xi^{\prime} /\left\|\xi^{\prime}\right\|$. Hence (if $b \Omega$ is connected) the number of zeros in each half plane does not depend on $\left(x^{\prime}, \omega^{\prime}\right)$. We let $\left\{\eta_{l}^{ \pm}\left(\omega^{\prime}\right): l=1, \ldots, L_{ \pm}\right\}$ denote the zeros of det $p_{0}\left(x^{\prime}, 0, \omega^{\prime}, \xi_{n}\right)$ in the upper (lower) half $\xi_{n}$-plane. The zeros may also depend on $x^{\prime}$, but we suppress that dependence for the time being. Evidently the sets

$$
Z_{ \pm}=\bigcup_{\omega^{\prime} \in S^{n-1}}\left\{\eta_{l}^{ \pm}\left(\omega^{\prime}\right): l=1, \ldots, L_{ \pm}\right\}
$$

have compact closures disjoint from the real axis.

Let $\Lambda_{ \pm}$be an interval on the real axis along with a semi-circle in $\pm \operatorname{Im} \xi_{n}>0$, enclosing $\overline{Z_{ \pm}}$. If $R>0$, then $R \Lambda_{ \pm}$denotes the contour scaled by the factor $R$. As an oscillatory integral we see that, for $\pm x_{n}>0$, we have

$$
\begin{aligned}
& \frac{1}{(2 \pi)^{n}} \int_{\mathbb{R}^{n-1}} \int_{-\infty}^{\infty} q_{j}\left(x^{\prime}, x_{n}, \xi^{\prime}, \xi_{n}\right) e^{i x_{n} \xi_{n}} d \xi_{n} \hat{g}\left(\xi^{\prime}\right) \psi\left(\xi^{\prime}\right) e^{i x^{\prime} \cdot \xi^{\prime}} d \xi^{\prime}= \\
& \quad \frac{1}{(2 \pi)^{n}} \int_{\mathbb{R}^{n-1}}\left[\int_{\left\|\xi^{\prime}\right\| \Lambda_{ \pm}} q_{j}\left(x^{\prime}, x_{n}, \xi^{\prime}, \xi_{n}\right) e^{i x_{n} \xi_{n}} d \xi_{n}\right] \hat{g}\left(\xi^{\prime}\right) \psi\left(\xi^{\prime}\right) e^{i x^{\prime} \cdot \xi^{\prime}} d \xi^{\prime} .
\end{aligned}
$$


It is not difficult to see that, for $\xi^{\prime} \neq 0$, the limits as $x_{n} \rightarrow 0^{ \pm}$exist, and define homogeneous symbols of degree $-j$ :

$$
\widetilde{q}_{ \pm j}\left(x^{\prime}, \xi^{\prime}\right)=\frac{1}{2 \pi} \int_{\left\|\xi^{\prime}\right\| \Lambda_{ \pm}} q_{j}\left(x^{\prime}, 0, \xi^{\prime}, \xi_{n}\right) d \xi_{n}
$$

For each $N \in \mathbb{N}$ we can also consider the remainder term:

$$
\begin{aligned}
R_{ \pm}^{N} g=\lim _{x_{n} \rightarrow 0^{ \pm}} \frac{1}{(2 \pi)^{n}} \int_{\mathbb{B}^{n-1}} \int_{-\infty}^{\infty}\left[q\left(x^{\prime}, x_{n}, \xi^{\prime}, \xi_{n}\right)-\sum_{j=0}^{N} q_{j}\left(x^{\prime}, x_{n}, \xi^{\prime}, \xi_{n}\right)\right] \\
\times \hat{g}\left(\xi^{\prime}\right) \psi\left(\xi^{\prime}\right) e^{i x_{n} \xi_{n}} d \xi_{n} e^{i x^{\prime} \cdot \xi^{\prime}} d \xi^{\prime}
\end{aligned}
$$

For large enough $N$, we can simply set $x_{n}=0$ and obtain an absolutely convergent integral giving a symbol of order $-1-N$ on $b \Omega$ :

$$
r^{N}\left(x^{\prime}, \xi^{\prime}\right)=\frac{1}{2 \pi} \int_{\mathbb{R}^{n-1}} \int_{-\infty}^{\infty}\left[q\left(x^{\prime}, 0, \xi^{\prime}, \xi_{n}\right)-\sum_{j=0}^{N} q_{j}\left(x^{\prime}, 0, \xi^{\prime}, \xi_{n}\right)\right] d \xi_{n} \psi\left(\xi^{\prime}\right)
$$

This shows that $\mathscr{P}_{ \pm}$are classical pseudodifferential operators of order 0 , with symbols $\widetilde{q}_{ \pm}$ satisfying

$$
\widetilde{q}_{ \pm}\left(x^{\prime}, \xi^{\prime}\right) \sim \sum_{j=0}^{\infty} \widetilde{q}_{ \pm j}\left(x^{\prime}, \xi^{\prime}\right) \sigma_{0}(P, \pm d r) .
$$

We now carry out the detailed computation of the principal symbol, which is homogeneous of degree zero. For each $\omega^{\prime} \in S^{n-2}$ we let $M_{ \pm}\left(x^{\prime}, \omega^{\prime}\right)$ denote the span of generalized nullspaces of

$$
\left\{p_{0}\left(x^{\prime}, 0, \omega^{\prime}, \eta_{l}^{ \pm}\left(\omega^{\prime}, x^{\prime}\right)\right): l=1, \ldots, L_{ \pm}\right\} .
$$

The fiber $E_{\left(x^{\prime}, 0\right)}$ is the direct sum $M_{+}\left(\omega^{\prime}, x^{\prime}\right) \oplus M_{-}\left(\omega^{\prime}, x^{\prime}\right)$. The subspaces $M_{ \pm}\left(\omega^{\prime}, x^{\prime}\right)$ consists of directions $v$ such that the system of ODEs:

$$
\begin{aligned}
& p_{0}\left(x^{\prime}, 0, \omega^{\prime}, \partial_{x_{n}}\right) v\left(x_{n}\right)=0 \\
& v(0)=v,
\end{aligned}
$$

has a solution, which is exponentially decaying as $\pm x_{n} \rightarrow \infty$. The principal symbol of $Q$ is $\left[p_{0}(x, \xi)\right]^{-1}$ and therefore, up a constant of modulus 1 ,

$$
\widetilde{q}_{ \pm 0}\left(x^{\prime}, \omega^{\prime}\right)=\frac{1}{2 \pi} \int_{\Lambda_{ \pm}}\left[p_{0}\left(x^{\prime}, 0, \omega^{\prime}, \xi_{n}\right)\right]^{-1} d \xi_{n} .
$$

are easily seen to be projections onto $M_{ \pm}\left(x^{\prime}, \omega^{\prime}\right)$, along $M_{\mp}\left(x^{\prime}, \omega^{\prime}\right)$.

A good treatment of the Calderon projector, in the general case, can be found in [14]; the case of Dirac operators can be found in [5].

\section{Fredholm Boundary Value Problems for First Order Operators}

We now examine boundary value problems for the elliptic first order operator $P$, considered in the previous section. The domain of the maximal extension of $P$ as an unbounded operator on $L^{2}, \operatorname{Dom}_{m a x}(P)$, consists of $L^{2}$-sections $u$ of $E \rightarrow \Omega$, such that the distributional derivative $P u$ is in $L^{2}$ as well. It follows from Corollary 2 that if $u \in \operatorname{Dom}_{\text {max }}(P)$, then $u$ has distributional boundary values in $H^{-\frac{1}{2}}(b \Omega)$. Hence, if $\mathscr{R}$ is 
a pseudodifferential operator acting on sections of $E \uparrow_{b \Omega}$, then we can define the domain of a closed, unbounded operator acting on $L^{2}(\Omega)$, by

$$
\operatorname{Dom}(P, \mathscr{R})=\left\{u \in \operatorname{Dom}_{\text {max }}(P): \mathscr{R}\left(u \uparrow_{b \Omega}\right)=0\right\} .
$$

We use the notation $(P, \mathscr{R})$ to denote this unbounded operator acting on $L^{2}(\Omega)$.

In this section we consider boundary conditions defined by pseudodifferential projections. This is not a serious restriction, since the nullspace, $\mathcal{N}_{\mathscr{R}}$, of $\mathscr{R}$ is a closed subspace. Under fairly mild conditions, (for example: 0 is isolated in the spectrum of $\mathscr{R}$ ), the orthogonal projection, $\mathscr{R}_{\mathrm{pr}}$, onto $\mathcal{N}_{\mathscr{R}}$ is a pseudodifferential operator. Evidently $(P, \mathscr{R})$ and $\left(P, \mathscr{R}_{\mathrm{pr}}\right)$ are the same operator on $L^{2}$. It is not necessary to assume that $\mathscr{R}$ is a classical pseudodifferential operator, but merely that it acts on $\mathscr{D}^{\prime}(b \Omega)$. We give a condition on $\mathscr{R}$ that insures that $(P, \mathscr{R})$ is a Fredholm operator.

As in the example of $\bar{\partial}$ on $D_{1}$, our analysis centers on the comparison operator. We let $\mathscr{P}$ denote the Calderon projector for $P$ on $\Omega$. If $\mathscr{R}$ is a projector defining a boundary condition for $P$, then we consider the operator:

$$
\mathscr{T}=\mathscr{R} \mathscr{P}+(\mathrm{Id}-\mathscr{R})(\mathrm{Id}-\mathscr{P}) .
$$

Assuming that $\mathscr{R}: H^{s}(b \Omega) \rightarrow H^{s}(b \Omega)$ for all $s \geq-\frac{1}{2}$, it follows from the fact that $\mathscr{P}$ is a classical pseudodifferential operator of order 0 , that $\mathscr{T}$ preserves the same Sobolev spaces.

Definition 4 We say that $\mathscr{R}$ is $\mu$-elliptic if $\mathscr{T}$ has parametrix $U$, for which there exists a $\mu \in \mathbb{R}$, such that for every $s \geq-\frac{1}{2}$,

$$
u: H^{s}(b \Omega) \rightarrow H^{s-\mu}(b \Omega),
$$

boundedly.

In this case we can select $U$ so that

$$
\text { UT }=\mathrm{Id}-K_{1} \text { and } \mathscr{T} U=\mathrm{Id}-K_{2},
$$

where $K_{1}, K_{2}$ are finite rank, smoothing operators.

The classical elliptic case corresponds to $\mu=0$. A small modification of the $\bar{\partial}$ Neumann condition on a strictly pseudoconvex, almost complex manifold gives an example where $\mu=\frac{1}{2}$, see $[9,8,10]$.

Theorem 11 Let $\Omega$ be a smooth manifold with boundary and $P: \mathscr{C}^{\infty}(\Omega ; E) \rightarrow$ $\mathscr{C}^{\infty}(\Omega ; F)$ a first order elliptic differential operator, with fundamental solution $Q$. Suppose that $\Re$ is a pseudodifferential projection acting on sections of $E \uparrow_{b \Omega}$. If $\Re$ is $\mu$-elliptic, with $\mu \leq 1$, then $(P, \mathscr{R})$ is a Fredholm operator; if $\mu<1$, then the unit ball in the domain of the operator, with respect to the graph norm, is compact in $L^{2}$. If the resolvent set is non-empty, then the operator has a compact resolvent.

Before proceeding with the proof of this theorem we observe that Lemma 1 has the following generalization:

Lemma 4 If $\mathscr{T} f \in \operatorname{Im} \mathscr{R}$ then $\mathscr{T} \mathscr{P} f=\mathscr{T} f$.

Proof This follows immediately from the fact that $\mathscr{R} \mathscr{T}=\mathscr{T} \mathscr{P}$.

Proof of the Theorem First we observe that $(P, \mathscr{R})$ has a finite dimensional nullspace. Suppose that $u \in \operatorname{Dom}(P, \mathscr{R})$ and $P u=0$. Corollary 2 implies that $u$ has distributional boundary values in $H^{-\frac{1}{2}}(b \Omega)$, which therefore satisfy $\mathscr{R}\left(u \uparrow_{b \Omega}\right)=0$. Since $u \in \operatorname{ker} P$, it is clear that $\mathscr{P}\left(u\left\lceil_{b \Omega}\right)=u\left\lceil_{b \Omega}\right.\right.$. Hence

$$
\mathscr{R}\left(u \uparrow_{b \Omega}\right)=\mathscr{T}\left(u\left\lceil_{b \Omega}\right)=0 .\right.
$$


On the other hand (140) then implies that

$$
\left(\mathrm{Id}-K_{1}\right) u \uparrow_{b \Omega}=0 .
$$

As $K_{1}$ is a smoothing operator, the nullspace of $\left(\mathrm{Id}-K_{1}\right)$ is finite dimensional. The existence of the fundamental solution $Q$ easily implies that elements of ker $P$ are determined by their boundary values on $b \Omega$. This shows that the nullspace of $(P, \mathscr{R})$ is finite dimensional.

Now we turn to the proof that the range is of finite codimension, and therefore closed. Let $f \in L^{2}(\Omega, F)$, and $u_{1}=Q f$, where, as usual, we extend $f$, by zero, to all of $\widetilde{\Omega}$, and

$$
u_{0}=-Q \sigma(P, d r)\left[\mathcal{R}\left(u_{1} \uparrow_{b \Omega}\right) \otimes \delta(r)\right] .
$$

We need to show that $u=u_{0}+u_{1} \in \operatorname{Dom}(P, \mathscr{R})$. That $P u=f$, in the sense of distributions, is clear. From Theorem 9 it follows that $u_{1} \in H^{1}(\Omega)$, and therefore $\mathcal{R}\left(u_{1}\lceil b \Omega\right.$ )$\in H^{\frac{1}{2}-\mu}(b \Omega)$. Hence Theorem 10 and the embedding result $H_{(1,-\mu)}(\Omega) \subset H^{1-\mu}(\Omega)$, imply that $u_{0} \in H^{1-\mu}(\Omega)$. If $\mu \leq 1$, then $u \in L^{2}(\Omega)$. To complete the argument, we need to show that $\mathscr{R}\left(u\left\lceil_{b \Omega}\right)=0\right.$. This is true, provided that $f$ satisfies finitely many bounded linear conditions.

We note that

$$
\mathscr{T} U \mathscr{R}\left(u_{1} \uparrow_{b \Omega}\right)=\left(\mathrm{Id}-K_{2}\right) \mathscr{R}\left(u_{1} \uparrow_{b \Omega}\right) .
$$

Recall that $K_{2}$ is of finite rank, hence the requirement

$$
K_{2}\left(\mathscr{R}\left(u_{1} \uparrow_{b \Omega}\right)\right)=K_{2} \mathscr{R}\left(Q f \uparrow_{b \Omega}\right)=0
$$

is a finite set of linear conditions on $f$. As the map $f \mapsto Q f \uparrow_{b \Omega}$ is bounded from $L^{2}(\Omega)$ to $H^{\frac{1}{2}}(b \Omega)$, these are evidently defined by bounded linear functionals. Let $S$ denote the subset of $L^{2}(\Omega ; F)$ where these conditions are satisfied. This is clearly a closed subspace of finite codimension.

If $f \in S$, then (144) and (145) imply that $\mathscr{T} \mathscr{R}\left(u_{1} \uparrow_{b \Omega}\right) \in \operatorname{Im} \mathscr{R}$. Lemma 4 then implies that

$$
\begin{aligned}
& \mathscr{R}\left(u_{0}\left\lceil_{b \Omega}\right)=-\mathscr{R} \mathscr{P} u \mathscr{R}\left(u_{1}\right\rceil_{b \Omega}\right) \\
& =-\mathscr{T} \mathscr{P} u \mathscr{R}\left(u_{1}\left\lceil_{b \Omega}\right)\right. \\
& =-\mathscr{T} U \mathscr{R}\left(u_{1}\lceil b \Omega)\right. \\
& =-\mathscr{R}\left(u_{1}\lceil b \Omega)\right. \text {. }
\end{aligned}
$$

To pass to the final line we use (145). Thus, if $f \in S$, then $\mathscr{R}\left(u \uparrow_{b \Omega}\right)=0$, and therefore $S$ is a subspace of the range of $(P, \mathscr{R})$. Hence the range of the operator is itself of finite codimension and therefore it is closed. This completes the proof that $(P, \mathscr{R})$ is a Fredholm operator provided $\mu \leq 1$.

Suppose that $u \in \operatorname{Dom}(P, \mathscr{R})$ and let $u_{1}=Q P(u) \in H^{1}(\Omega)$. The difference, $u-u_{1}$ is in the (formal) nullspace of $P$, hence

$$
\mathscr{P}\left(u-u_{1}\right) \uparrow_{b \Omega}=\left(u-u_{1}\right) \uparrow_{b \Omega} \text { and } \mathscr{R}\left(u-u_{1}\right) \uparrow_{b \Omega}=-\mathscr{R}\left(u_{1} \uparrow_{b \Omega}\right) .
$$

A priori, $\left(u-u_{1}\right)\left\lceil_{b \Omega} \in H^{-\frac{1}{2}}(b \Omega)\right.$. The identities in (147) imply that

$$
\mathscr{T}\left(u-u_{1}\right) \uparrow_{b \Omega}=-\mathscr{R}\left(u_{1} \uparrow_{b \Omega}\right) \in H^{\frac{1}{2}}(b \Omega) .
$$

Applying $u$, we see that

$$
\left(\operatorname{Id}-K_{1}\right)\left(u-u_{1}\right) \uparrow_{b \Omega}=-\vartheta \mathscr{R}\left(u_{1} \uparrow_{b \Omega}\right) \in H^{\frac{1}{2}-\mu}(b \Omega) .
$$

As $K_{1}$ is a smoothing operator, this shows that $\left(u-u_{1}\right) \uparrow_{b \Omega} \in H^{\frac{1}{2}-\mu}(b \Omega)$. Theorem 10 implies that $u-u_{1} \in H^{1-\mu}(\Omega)$ and therefore $u$ is as well. Thus the domain of $(P, \mathscr{R})$ is 
contained in $H^{1-\mu}(\Omega ; E)$. If $\mu<1$, then $H^{1-\mu}$ is compactly embedded into $L^{2}$, showing that the unit ball in the domain of the operator, with respect to the graph norm is compact in $L^{2}$. If ther resolvent set is non-empty, then the resolvent of $(P, \mathscr{R})$ is a compact operator. This completes the proof of the theorem.

Using the same argument we can also prove higher norm estimates.

Theorem 12 Under the hypotheses of Theorem 11, if $f \in H^{s}(\Omega ; F)$ satisfies finitely many linear conditions, then there exists a solution $u$ to

$$
P u=f \text { and } \mathscr{R}\left(u\left\lceil_{b \Omega}\right)=0 .\right.
$$

For each $s \geq 0$ there is a $C_{s}$ such that this solution satisfies the estimate

$$
\|u\|_{H^{s+1-\mu}} \leq C_{s}\|f\|_{H^{s}} .
$$

For $s \geq 0$, there is a $C_{s}^{\prime}$ such that if $u \in L^{2}, \mathscr{R}\left(u \uparrow_{b \Omega}\right)=0$, and $P u \in H^{s}(\Omega)$ then $u \in H^{s+1-\mu}(\Omega)$ and

$$
\|u\|_{H^{s+1-\mu}} \leq C_{s}^{\prime}\left[\|P u\|_{H^{s}}+\|u\|_{L^{2}}\right] .
$$

Proof We prove the last statement. If $u_{1}=Q P u$, then $u_{1} \in H^{s+1}(\Omega)$. Moreover, $P\left(u-u_{1}\right)=0$ and

$$
\mathscr{T}\left(u-u_{1}\right)\left\lceil_{b \Omega}=\mathscr{R}\left(u-u_{1}\right)\left\lceil_{b \Omega}=-\mathscr{R} u_{1}\left\lceil_{b \Omega} .\right.\right.\right.
$$

Hence

$$
\left(u-u_{1}\right)\left\lceil_{b \Omega}=-\mathcal{R} u_{1}\left\lceil_{b \Omega}+K_{1}\left(u-u_{1}\right)\left\lceil_{b \Omega} .\right.\right.\right.
$$

Since $u=\left(u-u_{1}\right)+Q P u$,

$$
\left(u-u_{1}\right)=Q\left(\sigma(P, d r)\left[\left(u-u_{1}\right)\left\lceil_{b \Omega}\right] \otimes \delta(r)\right),\right.
$$

and $K_{1}$ is smoothing operator, we easily obtain that there is a constant $C_{s}^{\prime}$ so that (152) holds.

The analysis of $(P, \mathscr{R})$ also leads immediately to an analysis of the Dirichlet problem for $P$ : given $f \in H^{s}(\Omega)$ and $g \in H^{s+\frac{1}{2}}(b \Omega) \cap \operatorname{Im} \Re$ find $u \in H^{s+1-\mu}(\Omega)$ such that:

$$
P u=f \text { and } \mathscr{R}\left(u \uparrow_{b \Omega}\right)=g .
$$

The analysis above shows that if $(f, g)$ satisfies finitely many bounded linear conditions $\ell_{i}(f, g)=0, i=1, \ldots, N$, then a solution to this problem exists. The dimension of the solution space equals the dimension of the nullspace of $(P, \mathscr{R})$.

If $\mathscr{R}$ is a classical pseudodifferential operator, then we can easily give symbolic conditions for $\mathscr{R}$ to be 0 -elliptic operator. The conditions are that for every $\left(x^{\prime}, \xi^{\prime}\right) \in T^{*} b \Omega \backslash\{0\}$, the restrictions

$$
\sigma_{0}(\mathscr{R})\left(x^{\prime}, \xi^{\prime}\right) \int_{\operatorname{Im} \sigma_{0}(\mathscr{P})\left(x^{\prime}, \xi^{\prime}\right)} \text { and }\left(\operatorname{Id}-\sigma_{0}(\mathscr{R})\left(x^{\prime}, \xi^{\prime}\right)\right) \prod_{\operatorname{Im}\left(\operatorname{Id}-\sigma_{0}(\mathscr{P})\left(x^{\prime}, \xi^{\prime}\right)\right)},
$$

are injective. This of course implies that $\sigma_{0}(\mathscr{T})\left(x^{\prime}, \xi^{\prime}\right)$ is invertible away from the zero section. If the projections are orthogonal, then $\sigma_{0}(\mathscr{R})\left(x^{\prime}, \xi^{\prime}\right)\left\lceil\operatorname{Im} \sigma_{0}(\mathscr{P})\left(x^{\prime}, \xi^{\prime}\right)\right.$ gives an isomorphism onto the image of $\sigma_{0}(\mathscr{R})\left(x^{\prime}, \xi^{\prime}\right)$ if and only if the complementary restriction $\left(\operatorname{Id}-\sigma_{0}(\mathscr{R})\left(x^{\prime}, \xi^{\prime}\right)\right) \uparrow_{\operatorname{Im}\left(\operatorname{Id}-\sigma_{0}(\mathscr{P})\left(x^{\prime}, \xi^{\prime}\right)\right)}$ gives an isomorphism onto the orthogonal complement $\operatorname{Im}\left(\operatorname{Id}-\sigma_{0}(\mathscr{R})\left(x^{\prime}, \xi^{\prime}\right)\right)$. 
Example 2 The most important example of an elliptic boundary condition for a first order system arises in the work of Atiyah, Patodi and Singer. In earlier work, Atiyah and Bott showed that there is a topological obstruction to the existence of a local, elliptic boundary condition for the Dirac operator on an even dimensional manifold, see [6]. Nonetheless, we can define elliptic boundary conditions using pseudodifferential projections. In this context one usually supposes that the Riemannian metric, $g$, reduces to a product in a neighborhood of $b \Omega$, so that a neighborhood of $b \Omega$ is foliated by totally geodesic hypersurfaces "parallel" to $b \Omega$. We let $r$ denote a coordinate labeling these hypersurfaces, with $\|d r\|_{g}=1$, in the collar neighborhood. If $\Omega$ is an even dimensional spin or $\operatorname{spin}_{\mathbb{C}}$ manifold, then the chiral Dirac operator, $P$ is an elliptic, first order operator, mapping sections of the bundle of even spinors to the bundle of odd spinors. We let $\boldsymbol{c}(d r)$ denote Clifford multiplication by the 1 -form $d r$; it defines unitary isomorphisms between the bundles of even and odd spinors. In a neighborhood of $b \Omega$, the Dirac operator takes the form:

$$
P=\boldsymbol{c}(d r)\left[\partial_{r}+B\right],
$$

where $B$ is a self adjoint operator acting on sections of the spin-bundle over $b \Omega$. The spinbundle over the boundary is isomorphic to the bundle of even (or odd) spinors restricted to the boundary. Since $b \Omega$ is compact without boundary, the operator $B$ has a pure point spectrum extending to $\pm \infty$. We let $\mathscr{R}$ denote the orthogonal projection onto the span of the eigenspaces of $B$, with non-negative eigenvalues. This is a classical pseudodifferential projection, which has the same principal symbol as the Calderon projector. Hence $\mathscr{R}$ is 0 -elliptic. It defines the Atiyah-Patodi-Singer boundary condition.

Example 3 As noted above, it is not necessary for $\mathscr{R}$ to be a classical pseudodifferential operator. In a series of papers, $[9,8,10,7]$, the case of a strictly pseudoconvex, Spin $_{\mathbb{C}}$-manifold is analyzed. In this context, a modification of the $\bar{\partial}$-Neumann condition can be defined that gives a $\frac{1}{2}$-elliptic operator. In this case, the boundary projection $\mathscr{R}$ belongs to the Heisenberg algebra defined by the contact structure on the boundary. The operator $U$ is a graded elliptic operator in the extended Heisenberg calculus.

While the integrability of the complex structure is not necessary for our analysis of boundary conditions for $\partial$, we restrict our attention to the case of a compact Kähler manifold, $\Omega$, with a strictly pseudoconvex boundary. The usual convention is to use a defining function $\rho$ for $b \Omega$, which is negative in int $\Omega$. Strict pseudoconvexity is the condition that, for large enough $\lambda$, the complex Hessian, $\partial \bar{\partial} e^{\lambda \rho}$, is positive definite along $b \Omega$. The one form $\theta=i \bar{\partial} \rho\left\lceil_{b \Omega}\right.$ is real; the strict pseudoconvexity of the boundary implies that subbundle $H=\operatorname{ker} \theta$ of $T b \Omega$ is a contact structure. There is a special calculus of pseudodifferential operators, $\Psi_{H}^{*}$, called the Heisenberg calculus defined on a contact manifold, see $[4,21]$. This calculus plays an essential role in the analysis of the $\bar{\partial}$-operator. A strictly pseudoconvex manifold has an infinite dimensional space of holomorphic functions. As in the 1-dimensional case, a holomorphic function is determined by its boundary values on $b \Omega$. We let $\mathscr{S}$ denote the Szegó projector. This is an orthogonal projection operator with range equal to the set of boundary values of holomorphic functions on $\Omega$. If the complex dimension of $\Omega$ is greater than one, then the Szegó projector is not a classical pseudodifferential operator, but rather an element of $\Psi_{H}^{0}(b \Omega)$.

As noted in Example 1, the bundle of spinors on a complex manifold can be identified with $\$ \simeq \oplus \Lambda^{0, q} \Omega$. Thus a spinor can be decomposed into $(0, q)$-types:

$$
\sigma=\sigma^{0,0}+\sigma^{0,1}+\cdots+\sigma^{0, n} .
$$


The $\bar{\partial}$-Neumann condition for $(0, q)$-forms is the requirement that

$$
\left.[\bar{\partial} \rho\rfloor \sigma^{0, q}\right]\left\lceil_{b \Omega}=0 .\right.
$$

A moments thought shows that this does not impose any condition on $(0,0)$-forms, and for this reason, does not lead to a Fredholm boundary value problem for $\bar{\partial}=\bar{\partial}+\bar{\partial}^{*}$ acting on sections of $\$$. Indeed, any holomorphic function, $f$ satisfies $\partial f=0$ and the boundary condition (160). This situation admits of a simple remedy: we use the condition in (160) for $2 \leq q \leq n$. For $q=0$ we impose the condition:

$$
\mathscr{S}\left[\sigma^{0,0} \uparrow_{b \Omega}\right]=0 .
$$

In order for the boundary value problem for $ð$ to be formally self adjoint, we need to modify the boundary condition for $q=1$ to

$$
\left.(\operatorname{Id}-\mathscr{Y})[\bar{\partial} \rho\rfloor \sigma^{0,1} \Gamma_{b \Omega}\right]=0 .
$$

This boundary condition is defined by the projector $\mathscr{R}$ :

$$
\mathscr{R}=\left(\begin{array}{ccccc}
\mathscr{S} & 0 & 0 & \ldots & 0 \\
0 & (\mathrm{Id}-\mathscr{S}) \bar{\partial} \rho\rfloor & 0 & \ldots & 0 \\
0 & 0 & \bar{\partial} \rho\rfloor & \ldots & 0 \\
\vdots & \vdots & \vdots & \ddots & \vdots \\
0 & 0 & 0 & \ldots & \bar{\partial} \rho\rfloor
\end{array}\right),
$$

acting on sections of $\$ \uparrow_{b \Omega}$.

If we let $\mathscr{P}$ be the Calderon projector for $ð$, then $\mathscr{T}=\mathscr{R} \mathscr{P}+(\operatorname{Id}-\mathscr{R})(\operatorname{Id}-\mathscr{P})$. In $[9,8$, $10,7]$ it is shown that the operator $\mathscr{R}$ is $\frac{1}{2}$-elliptic; that is: $\mathscr{T}$ has a parametrix, $\mathscr{U}$, such that $q: H^{s} \rightarrow H^{s-\frac{1}{2}}$ for $s \in \mathbb{R}$. This result is proved using the extended Heisenberg calculus. The analysis is closely related to the analysis of the $\bar{\partial}$-Neumann problem on $(0,1)$-forms given by Greiner and Stein in [12].

As in Example 1, the operator $(ð, \mathscr{R})$ is self adjoint, but can be split into its chiral parts $\left(\check{\partial}^{\mathrm{eo}}, \mathscr{R}^{\mathrm{eo}}\right)$. These operators are also Fredholm, moreover,

$$
\left(\check{\partial}^{\mathrm{eo}}, \Re^{\mathrm{eo}}\right)^{*}=\left(\check{\partial}^{\mathrm{oe}}, \mathscr{R}^{\mathrm{oe}}\right) \text {. }
$$

In the case of a compact Kähler manifold with strictly pseudoconvex boundary, the index of $\left(\check{\partial}^{\mathrm{e}}, \mathscr{R}^{\mathrm{e}}\right)$ is nothing but the holomorphic Euler characteristic of $\Omega$, with the contribution of the infinite dimensional group $H^{0,0}(\Omega)$ removed:

$$
\operatorname{Ind}\left(\mathscr{\partial}^{\mathrm{e}}, \mathscr{R}^{\mathrm{e}}\right)=\sum_{q=1}^{n} \operatorname{dim} H^{0, q}(\Omega)(-1)^{q} .
$$

It is not necessary to use the classical Szegő projector in the definition of $\mathscr{R}$ in order to get a Fredholm boundary value problem. Let $\mathscr{S}^{\prime}$ be a projector in $\Psi_{H}^{*}(b \Omega)$ that resembles, symbolically, a classical Szegó projector. If we let $\mathscr{R}^{\prime}$ denote the projector defined in equation (163), with $\mathscr{S}$ replaced by $\mathscr{S}^{\prime}$, then $\left(ð, \mathscr{R}^{\prime}\right)$ is again a Fredholm operator. The restriction

$$
\mathscr{S}: \operatorname{Im} \mathscr{S}^{\prime} \longrightarrow \operatorname{Im} \mathscr{Y}
$$

is also a Fredholm operator, we denotes its index by $\operatorname{R}-\operatorname{Ind}\left(\mathscr{S}^{\prime}, \mathscr{Y}\right)$. Generalizing a classical result of Agranovich and Dynin, one can show that

$$
\operatorname{Ind}(ð, \mathscr{R})-\operatorname{Ind}\left(ð, \mathscr{R}^{\prime}\right)=\operatorname{R}-\operatorname{Ind}\left(\mathscr{Y}^{\prime}, \mathscr{Y}\right) .
$$


While they are wildly different types of operators, there is even a sense in which $\mathscr{R}^{\prime}$ and $\mathscr{P}$ have a relative index, and indeed:

$$
\operatorname{Ind}\left(ð, \mathscr{R}^{\prime}\right)=\mathrm{R}-\operatorname{Ind}\left(\mathscr{P}, \mathscr{R}^{\prime}\right) .
$$

In fact, we can use this identity to express the index of $\left(ð, \mathscr{R}^{\prime}\right)$ in terms of a trace on the boundary:

$$
\operatorname{Ind}\left(ð, \mathscr{R}^{\prime}\right)=\operatorname{tr} \mathscr{P} K_{1} \mathscr{P}-\operatorname{tr} \mathscr{R}^{\prime} K_{2} \mathscr{R}^{\prime} .
$$

This follows, as before, from the fact that

$$
\left(\mathscr{P} u \mathscr{R}^{\prime}\right) \mathscr{R}^{\prime} \mathscr{P}=\mathscr{P}\left(\mathrm{Id}-K_{1}\right) \mathscr{P} \text { and } \mathscr{R}^{\prime} \mathscr{P}\left(\mathscr{P} u \mathscr{R}^{\prime}\right)=\mathscr{R}^{\prime}\left(\mathrm{Id}-K_{2}\right) \mathscr{R}^{\prime} .
$$

A careful discussion of these results would take us too far afield, details can be found in the papers cited above.

\section{References}

[1] M.F. Atiyah, V.K. Patodi, and I.M. Singer. Spectral asymmetry and Riemannian geometry, I. Math. Proc. Camb. Phil. Soc, 77:43-69, 1975.

[2] M.F. Atiyah, V.K. Patodi, and I.M. Singer. Spectral asymmetry and Riemannian geometry, II. Math. Proc. Camb. Phil. Soc, 78:405-432, 1975.

[3] M.F. Atiyah, V.K. Patodi, and I.M. Singer. Spectral asymmetry and Riemannian geometry, III. Math. Proc. Camb. Phil. Soc, 79:71-99, 1976.

[4] R. Beals and P. Greiner. Calculus on Heisenberg Manifolds, volume 119 of Annals of Mathematics Studies. Princeton University Press, 1988.

[5] B. Booss-Bavnbek and K. P. Wojciechowsi. Elliptic Boundary Problems for the Dirac Operator. Birkhäuser, Boston, 1996.

[6] M.F. Atiyah and Raoul Bott. The index theorem for manifolds with boundary. In Differential Analysis, Bombay Colloquium, pages 175-186. Oxford Press, 1964

[7] Charles L. Epstein. Subelliptic boundary conditions for $\operatorname{Spin}_{\mathbb{C}}$ Dirac operators, gluing, relative indices, and tame Fredholm pairs. Proc. Nat Acad. of Sci., 103:15364-15369, 2006.

[8] Charles L. Epstein. Subelliptic $\operatorname{Spin}_{\mathbb{C}}$ Dirac operators,I. to appear Annals of Math., pages 1-36, 2006.

[9] Charles L. Epstein. Subelliptic Spin $\mathbb{C}$ Dirac operators,II. to appear Annals of Math., pages 1-54, 2006.

[10] Charles L. Epstein. Subelliptic Spin $\mathbb{C}$ Dirac operators,III. to appear Annals of Math., pages 1-72, 2006.

[11] Charles L. Epstein. Pseudodifferential Methods for Boundary Value Problems. in Pseudo-Differential Operators: Partial Differential Equations and Time-Frequency Analysis, Luigi Rodino, Bert-Wolfgang Schulze, and M. W. Wong, editors. Fields Institute Communications, volume 52,AMS, Providence, RI. 414pp., 2007.

[12] P. C. Greiner and E. M. Stein. Estimates for the $\bar{\partial}$-Neumann problem. Mathematical Notes. Princeton University Press, 1977.

[13] L. Hörmander. The Analysis of Linear Partial Differential Operators, volume 1. Springer-Verlag, Berlin, Heidelberg, New York, Tokyo, 1983.

[14] L. Hörmander. The Analysis of Linear Partial Differential Operators, volume 3. Springer-Verlag, Berlin, Heidelberg, New York, Tokyo, 1985.

[15] Hyunsuk Kang. Polynomial Hulls and Relative Indices in Dimension One. Thesis. University of PA, Philadelphia, PA, 2003.

[16] Oliver Dimon Kellogg. Foundations of Potential Theory. Dover Publications, Inc., 1953.

[17] Peter D. Lax. Functional Analysis. Pure and Applied Mathematics. John Wiley and Sons, New York, 2002.

[18] Richard B. Melrose. Introduction to Microlocal Analysis. Lecture notes available at http://wwwmath.mit.edu/ rbm/iml90.pdf.

[19] R.T. Seeley. Singular integrals and boundary value problems. Amer. Jour. of Math., 88:781-809, 1966.

[20] Elias Stein. Singular Integrals and Differentiability Properties of Functions. Princeton Press, 1970.

[21] Michael E. Taylor. Noncommutative microlocal analysis, part I, volume 313 of Mem. Amer. Math. Soc. AMS, 1984.

[22] Michael E. Taylor. Partial Differential Equations, Vol. 2, volume 116 of Applied Mathematical Sciences. Springer, New York, 1996. 